Article

\title{
Light- and Temperature-Induced Expression of an R2R3-MYB Gene Regulates Anthocyanin Biosynthesis in Red-Fleshed Kiwifruit
}

\author{
Min Yu ${ }^{1,2}$, Yuping Man ${ }^{1}$ and Yanchang Wang ${ }^{1, *}$ \\ 1 Key Laboratory of Plant Germplasm Enhancement and Specialty Agriculture, Wuhan Botanical Garden, \\ Chinese Academy of Sciences, Wuhan 430074, China; yumindsd@163.com (M.Y.); \\ manyuping@126.com (Y.M.) \\ 2 College of Life Sciences, University of Chinese Academy of Sciences, Beijing 100049, China \\ * Correspondence: kiwifruit@wbgcas.cn; Tel.: +86-27-87700883; Fax: +86-27-87700826
}

Received: 18 August 2019; Accepted: 16 October 2019; Published: 22 October 2019

\begin{abstract}
The R2R3 MYB genes associated with the flavonoid/anthocyanidin pathway feature two repeats, and represent the most abundant classes of MYB genes in plants; however, the physiological role and regulatory function of most R2R3 MYBs remain poorly understood in kiwifruit (Actinidia). Here, genome-wide analysis identified 155 R2R3-MYBs in the 'Red $5^{\prime}$ version of the Actinidia chinensis genome. Out of 36 anthocyanin-related AccR2R3-MYBs, AcMYB10 was the most highly expressed in inner pericarp of red-fleshed kiwifruit. The expression of AcMYB10 was highly correlated with anthocyanin accumulation in natural pigmentation during fruit ripening and light-/temperature-induced pigmentation in the callus. AcMYB10 is localized in the nuclei and has transcriptional activation activity. Overexpression of $A c M Y B 10$ elevates anthocyanin accumulation in transgenic $A$. chinensis. In comparison, $A$. chinensis fruit infiltrated with virus-induced gene silencing showed delayed red coloration, lower anthocyanin content, and lower expression of AcMYB10. The transient expression experiment in Nicotiana tabacum leaves and Actinidia arguta fruit indicated the interaction of $A c M Y B 10$ with $A c b H L H 42$ might strongly activate anthocyanin biosynthesis by activating the transcription of AcLDOX and AcF3GT. In conclusion, this study provides novel molecular information about R2R3-MYBs in kiwifruit, advances our understanding of light- and temperature-induced anthocyanin accumulation, and demonstrates the important function of AcMYB10 in the biosynthesis of anthocyanin in kiwifruit.
\end{abstract}

Keywords: AcMYB10; kiwifruit; R2R3-MYB; anthocyanin; light; temperature

\section{Introduction}

MYB transcription factors (TFs) are found in a wide range of higher plants and constitute one of the largest transcription factor families. They are characterized by a structurally conserved DNA-binding domain that consists single or multiple imperfect repeats located near the $\mathrm{N}$-terminus. These repeats function synergistically or individually in DNA binding and protein-protein interactions, respectively [1]. In plants, MYB-containing genes have greatly diversified, being classified into four major subfamilies (1R-, R2R3-, 3R-, and 4R-MYB) based on the number of adjacent MYB repeats [2]. R2R3-MYB proteins probably evolved from an R1-MYB gene through the duplication of an R1 repeat or from an R1R2R3-MYB gene through the loss of an R1 repeat, and form the largest subfamily of MYB transcription factors in the MYB super family [2]. For example, 198 MYB genes were identified in Arabidopsis thaliana, of which 126 belong to R2R3-MYB [3]. Furthermore, 88 and 244 R2R3-MYB proteins have been identified in rice (Oryza sativa) and soybean (Glycine max), respectively [4,5]. R2R3-MYB proteins play an important role in regulatory networks that are involved in metabolic, cellular, and 
developmental processes, as well as responses to biotic and abiotic stresses [3]. The first plant MYB gene, C1, was isolated from maize (Zea mays), and encodes a c-MYB-like protein involved in the anthocyanin biosynthesis pathway [6]. More recently, R2R3-MYBs were reported to contribute to regulation of anthocyanin accumulation in Arabidopsis, grape (Vitis vinifera), Asian pear (Pyrus pyrifolia), and apple (Malus domestica) [7-10].

Kiwifruit, namely the genus Actinidia, is represented by 54 species and 75 taxa [11] and is one of the most valuable fruit crops worldwide $(5,287,605$ tons in 2014, http://www.fao.org/). Among the kiwifruit taxa, red pigmentation is the result of the accumulation of anthocyanins and only occurs in a few species, such as Actinidia chinensis, Actinidia arguta, Actinidia deliciosa, and Actinidia eriantha [12]. For this reason, red-fleshed kiwifruit cultivars, such as A. chinensis cv. Hongyang, have attracted increasing interest by consumers because of its excellent fruit quality and health properties attributed to anthocyanins $[13,14]$. The genes associated with anthocyanin metabolism in kiwifruit have been studied over the last decade. AcF3GT1 is a key structural gene in the anthocyanin biosynthesis pathway and is required for anthocyanin biosynthesis in red-fleshed A. chinensis [15]. R2R3-MYBs are also involved in the biosynthesis of anthocyanin in kiwifruit. AcMYB110a determines the red color of the petal and activates F3GT1 to induce anthocyanin accumulation in A. erientha [16]. In 'Hongyang', the expression of $A c M Y B 75$ is closely related to anthocyanin accumulation during fruit development [17]. Transient color assays reveal that $A c M Y B F 110$ can autonomously induce anthocyanin accumulation in N. tabacum leaves by activating the transcription of NtDFR, NtANS, and NtUFGT [18]. Using both transient assays in N. tabacum leaves or A. arguta fruits and stable transformation in Arabidopsis, a previous report demonstrated that co-expression of $A c M Y B 123$ and AcbHLH42 is a prerequisite for anthocyanin production by activating transcription of AcF3GT1 and AcANS [19].

Anthocyanin-related R2R3-MYB is closely regulated by environmental factors such as light and temperature [20]. In A. thaliana, anthocyanin biosynthesis is influenced by different light qualities through MYB (PAP1 and PAP2) and bHLH (TT8, EGL3, and GL3) [21]. Accumulation of anthocyanins in response to light is mediated by R2R3 MYB transcription factors in many fruit crops, including apple, pear, litchi, eggplant, and bayberry [22-26]. In addition to light, temperature is an important factor that affects anthocyanin biosynthesis in plants. High temperatures decrease anthocyanin content in the skin of apple and grapevine berries $[27,28]$. Light and temperature also have a synergistic effect on anthocyanin accumulation in fruit. For instance, in grape berry, low temperature and light intensity have a synergistic effect on the expression of genes in the flavonoid biosynthesis pathway in the skin [27]. In red-fleshed apple, light and temperature are important factors involved in the anthocyanin biosynthesis of callus cultures, by regulating the expression of MYBs [29]. In kiwifruit, the up-regulation of MYBA1-1 and MYB5-1 by low temperature could effectively enhance anthocyanin accumulation in kiwifruit during storage, probably through the transcriptional activation of ANS1, ANS2, DFR1, DFR2, and UFGT2 [30]. By contrast, high temperature suppresses AcMYB1 expression, which contributes to reduce anthocyanin accumulation in kiwifruit [31]. However, the molecular mechanisms underlying the combined effects of light and temperature on anthocyanin biosynthesis have not been comprehensively investigated in kiwifruit.

This study aimed to characterize the candidate R2R3-MYBs involved in the accumulation of anthocyanin in kiwifruit and to understand how the regulatory network of anthocyanin biosynthesis is affected by light and temperature. The results are expected to allow us to elucidate the function of the key MYBs responsible for anthocyanin accumulation in kiwifruit. Our findings are also expected to provide new insights on the functions of MYB genes in red-flesh kiwifruit, providing a baseline for detecting genes of significance for genetic manipulation. 


\section{Results and Discussion}

\subsection{Identification, Genomic Location, Gene Structure, and Motif Composition of R2R3-MYB Genes in Kiwifruit}

In total, 224 deduced amino acid sequences that contain MYB or MYB-Like domains were identified in the "Red5" kiwifruit genome. Among these, 155 R2R3-MYB (AccR2R3-MYB) were identified based on a conserved domain search, and proteins shared 108 conserved R2R3 residues. From the alignment, a series of periodic Trp (W) residues was found in each MYB repeat, which were considered as a landmark of the MYB domain. In addition to the highly conserved tryptophan residues, Asp-14, Cys-45, and Arg-48 in R2 repeat; Leu-53 in the linker region; and Arg-91 and Thr-92 in the R3 repeat were also completely conserved in all of the AccR2R3-MYB proteins (Supplementary Figure S1A).

The 155 AccR2R3-MYB genes were unevenly distributed across all 29 chromosomes, except chromosome 19 (Supplementary Figure S1B). Chromosome 23 harbored the most abundant (13) AccR2R3-MYBs, followed by chromosomes 14 and 18 (10). In comparison, only two AccR2R3-MYBs were found on chromosomes 2, 4, 6, 10, and 21, respectively (Supplementary Figure S1B).

To investigate the conserved motifs in this family, the 155 AccR2R3-MYBs were subjected to MEME Suite 5.0.5 (Supplementary Figure S2). All of these 155 AccR2R3-MYB contained 1\#, 2\#, and 3\# motifs, which, together, constructed the conserved R2R3 domain. Most of the phylogenetically close members had common conserved motifs, indicating that they had similar biological functions.

The genomic length of AccR2R3-MYB ranged from 970 bp (Acc27824) to 10,640 bp (Acc15993) (Supplementary Figure S2). 126 AccR2R3-MYB genes contained three exons, 25 contained two exons, three (Acc02688, Acc29660, and Acc14973) contained four exons, and only Acc27824 contained one exon. Most of these AccR2R3-MYBs contained one to two introns.

\subsection{Phylogenetic Analysis of AccR2R3-MYB TFs}

To subclassify the AccR2R3-MYBs, a neighbor-joining (NJ) phylogenetic tree was constructed using the R2R3 conserved domain of the 155 AccR2R3-MYBs and 126 AtR2R3-MYBs (Supplementary Figure S3). According to the tree topology, bootstrap values, and the previous classification of AtR2R3-MYBs [3,32], we classified the 155 AccR2R3-MYBs into 27 subgroups (designated C1-C27 in Supplementary Figure S3). The largest group (C16) contained 14 members, followed by C11, which contained 13 members. In comparison, group C27 was the smallest, containing only one AccR2R3-MYB member. Among the 27 groups, $C 9$ to C14 were in a same clade, containing AtMYB75, AtMYB90, AtMYB113, and AtMYB114 (highlighted in red in Supplementary Figure S3), which are involved in anthocyanin biosynthesis in Arabidopsis [7]. Acc10232, Acc00493, and Acc10227 had a very close phylogenetic relationship with these anthocyanin-related AtMYBs, indicating that their functions were related. More importantly, Acc10232 (AcMYB110) determined anthocyanin accumulation in the kiwifruit petal [16]. Thus, the 36 AccR2R3-MYBs in C9 to C14, especially Acc10232 (AcMYB110), Acc00493 (AcMYB10), and Acc10227, are probably involved in anthocyanin biosynthesis in kiwifruit.

\subsection{Expression Preference of AccR2R3-MYBs in Kiwifruit}

The expression preference of the identified AccR2R3-MYBs was investigated in four tissues, including the callus, leaf, flower, and inner pericarp using RNA-seq data (Supplementary Table S1). The expression levels of 155 R2R3-MYBs were clustered into four main groups, as shown in Supplementary Figure S3. Of note, all of the 155 AccR2R3-MYBs showed various tissue preferences, and their expression levels were not clustered in the phylogenetic tree (Supplementary Figure S4). Thus, the expression levels of the 36 anthocyanidin-related MYBs were exclusively clustered again (Figure 1A). In addition, only one gene, Acc00493 (AcMYB10), was clustered alone, and showed an expression preference for the inner pericarp of fruit, indicating that it is involved in the fruit pigmentation of kiwifruit. To verify the RNA-seq data, quantitative real-time RT-PCR was performed 
on four different tissues for 14 selected AccR2R3-MYBs. As shown in Figure 1B, the genes showed very distinct tissue-specific expression patterns, which were in good agreement with the RNA-seq data.

A

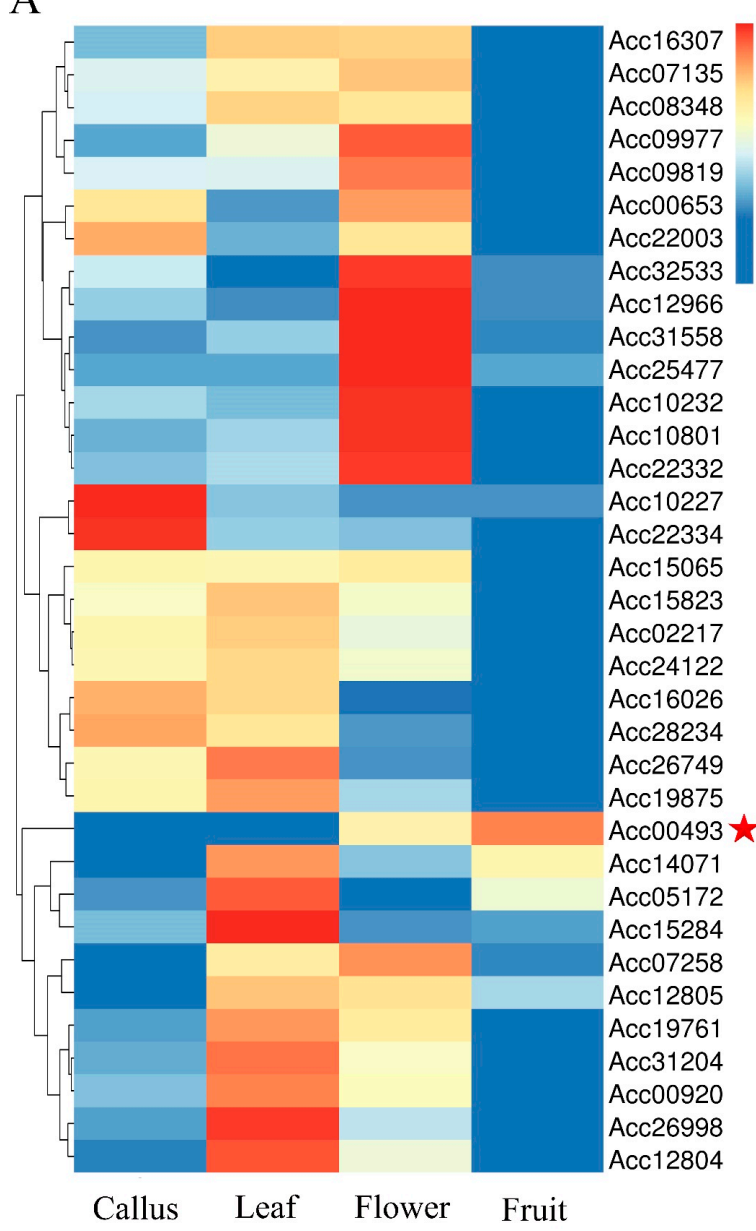

B
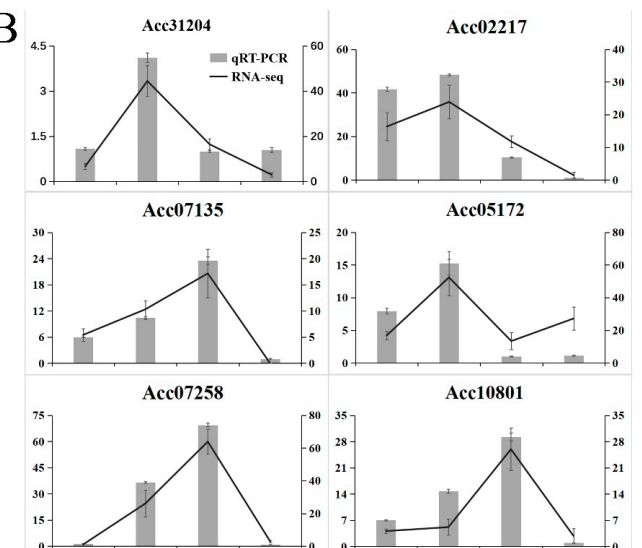

Acc00493 $\star$
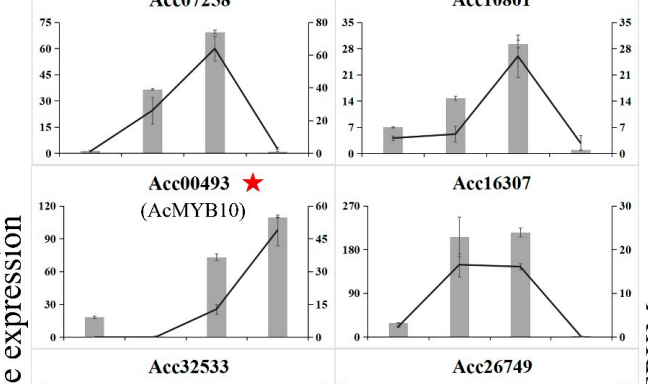

Acc16307
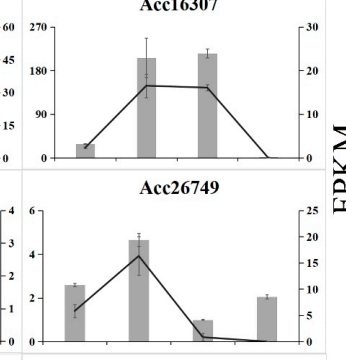

Acc15284

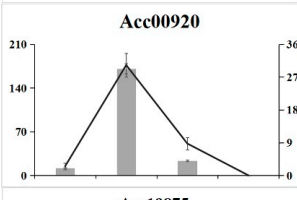

Acc19875

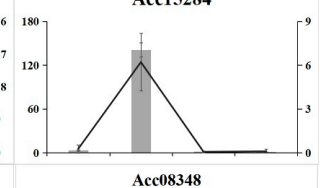

Acc08348

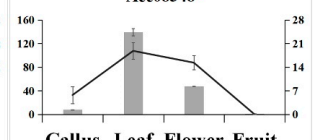

Figure 1. Tissue expression profiles of 36 anthocyanin-related AccR2R3-MYB genes in kiwifruit. (A) Hierarchical clustering of expression profiles of 36 candidate AccR2R3-MYBs in different tissues. Log2(FPKM+1) (Fragments per kilobase of transcript per million mapped reads) values were displayed according to the color code (top right). (B) Expression analysis of selected genes using quantitative real-time RT-PCR in different tissues. AcMYB10 (Acc00493) was marked with a red pentagram.

\subsection{Identification of Cis-Acting Regulatory Elements of Anthocyanin-Related AccR2R3-MYBs}

To establish the "regulation-expression" cue of these candidate AccR2R3-MYBs, the cis-acting regulatory elements (CREs) located at $2000 \mathrm{bp}$ upstream of the 36 genes (designated C1-C27 in Supplementary Figure S3) were identified using the PlantCARE database (Supplementary Table S2). A total of 3405 CREs were identified and classified into six groups based on their annotations, which included 'cellular regulation' (72.3\%), 'light responsiveness' (14.2\%), 'hormone signaling' (7.4\%), 'biotic and abiotic stresses' (4.0\%), 'developmental processes' (1.5\%), and 'biosynthetic processes' $(0.7 \%)$. In the 'light responsiveness' group, most of these CREs were allocated to 'G-Box' (127) and 'Box 4' (97). The 'biotic and abiotic stresses' group contained 135 CREs, of which 15 were allocated to low-temperature stress (LTR). The distribution of CREs involved in responsiveness and development is shown in Figure 2. 'Light responsiveness' was the dominant CRE that was distributed in all of the promoters. However, the distribution of the CRE in these promoters was highly diverse, but not conserved. Specifically, there was no parallel conservation that was correlated to the phylogenetic tree 
constructed based on the coding sequences (CDS) of the 36 anthocyanidin-related MYBs (Supplementary Figure S3). Thus, these MYBs have various regulatory patterns, indicating that they evolved quickly with high functional differentiation. This result supports the pattern of expression for these MYBs shown in Figure 1A. Thus, combined with previous studies on the regulation of MYBs correlated to anthocyanidin accumulation by environmental factors [20], we speculated that the expression of anthocyanin-related AccR2R3-MYBs would also respond to light or temperature in specific tissues.
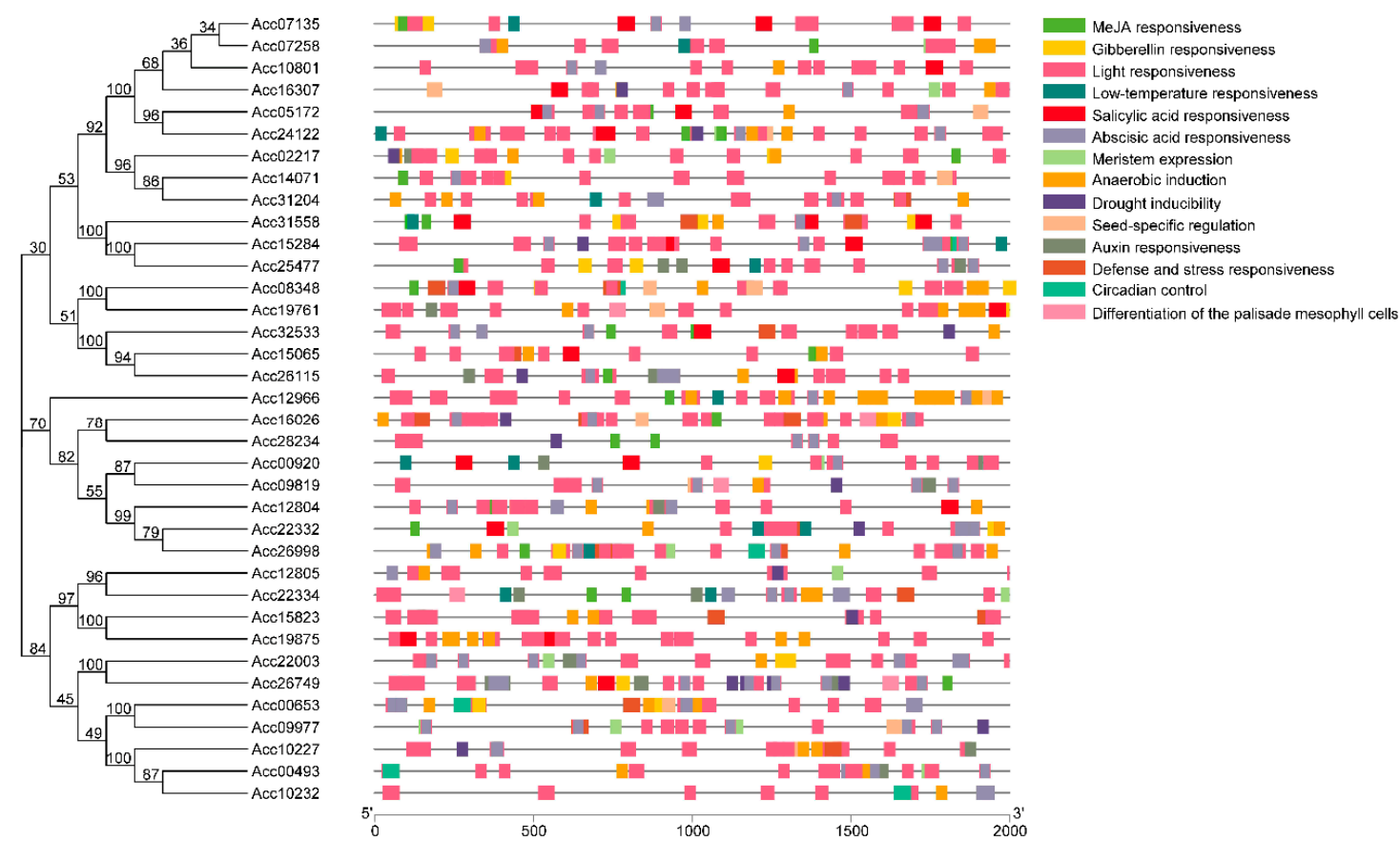

Figure 2. Distribution of cis-acting regulatory elements (CREs) involved in the responsiveness and development of the promoters of 36 candidate AccR2R3-MYBs.

\subsection{Light and Low-Temperature Synergistically Induced the Red Pigmentation in the Callus of}

A. chinensis cv. Hongyang

To investigate the effect of light and temperature on anthocyanin accumulation in kiwifruit, the callus of 'Hongyang' was cultured at different temperatures and light/dark conditions. As shown in Figure $3 \mathrm{~A}$, under the light-to-dark condition, the red pigment gradually accumulated in the $16{ }^{\circ} \mathrm{C}$ and $24^{\circ} \mathrm{C}$ treatments when cultured under days with continuous light conditions. Then, the redness of the callus gradually faded after transition to dark at $16^{\circ} \mathrm{C}$ and immediately faded at $24{ }^{\circ} \mathrm{C}$. Only a little pigment appeared on the callus at $32^{\circ} \mathrm{C}$ treatment under light or dark conditions. Under the dark-to-light condition, all the callus remained green/pale-green under continuous darkness. However, the callus immediately became red after exposure to light at $16^{\circ} \mathrm{C}$ and $24{ }^{\circ} \mathrm{C}$. In comparison, at $32{ }^{\circ} \mathrm{C}$, the callus showed no obvious color change during the whole process. Anthocyanin content was determined in all of these samples (Figure 3B). Variation in anthocyanin levels in every treatment of the callus was strongly consistent with the level of red pigmentation, thus leading to the anthocyanin biosynthesis of kiwifruit responses to light and low temperature. This suggestion supports previous reports about the synergistic effects of light and temperature on anthocyanin accumulation in grape skin and apple callus [27,29]. High temperature might inhibit (light-induced) anthocyanin biosynthesis or promote anthocyanin degradation in kiwifruit, as reported for grape [33]. 

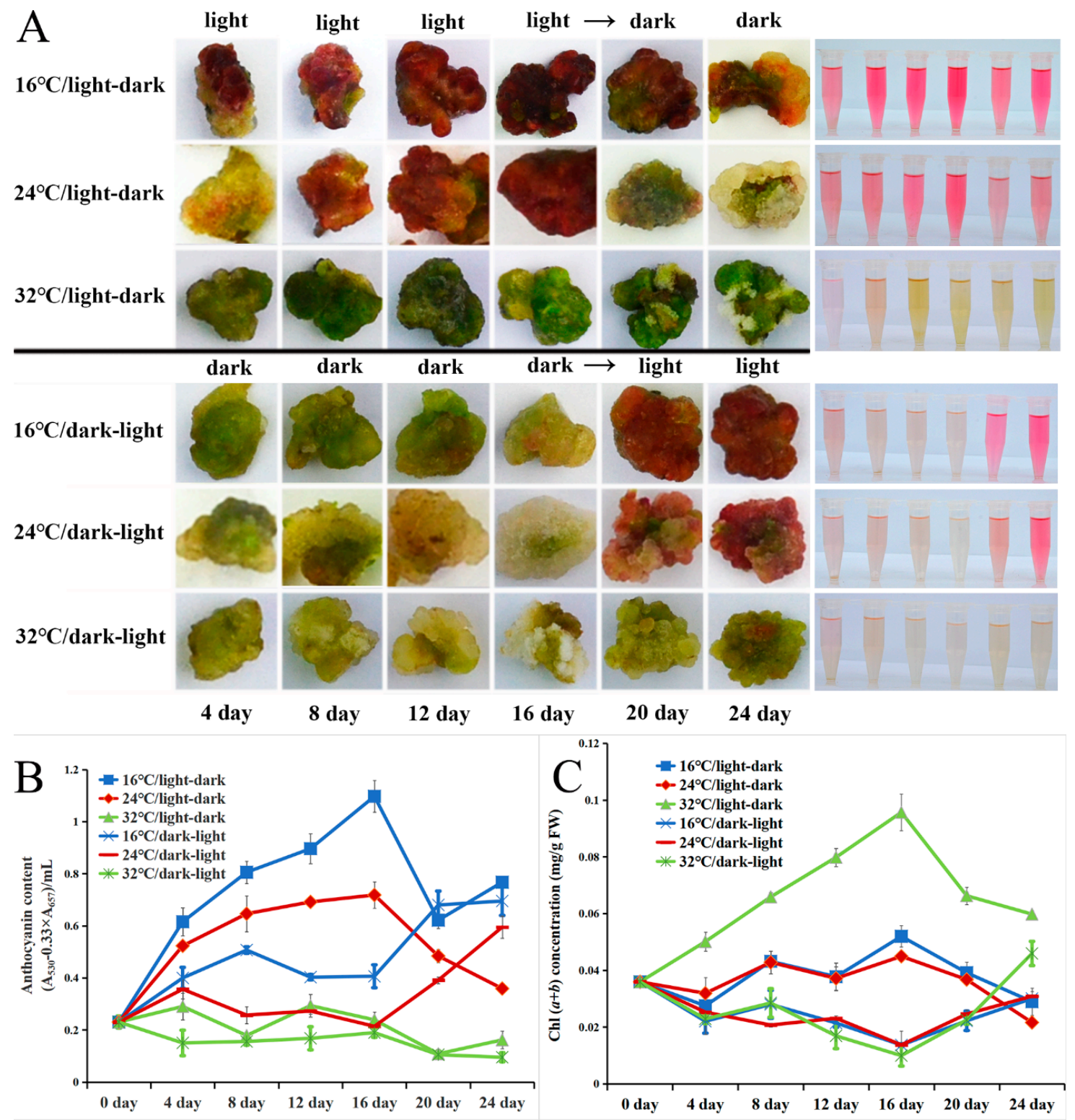

Figure 3. Relative callus anthocyanin content and chlorophyll content under different temperatures and light conditions. (A) Changes to callus color under different conditions and culture stages. The tubes in the right part contain the fresh extracts from left callus. (B) Anthocyanin content under different culture conditions over 24 days. (C) Chlorophyll ( $\mathrm{Chl} a+\mathrm{Chl} b)$ content over 24 days. Results represent the mean \pm SD of three replicates.

Of note, the green pigmentation changed regularly with treatment in our study (Figure 3A). Previous studies reported that the accumulation of both chlorophyll and anthocyanin is affected by light and temperature $[27,34]$, with the green pigment (chlorophyll) showing opposite accumulation trends to the red pigment (anthocyanin) during fruit ripening [35]. Thus, we investigated the chlorophyll content of all callus samples. As shown in Figure 3C, under the light-to-dark condition, chlorophyll content increased under continuous light and sharply decreased after light/dark transition, showing the same trend as anthocyanin content (except at $32{ }^{\circ} \mathrm{C}$ ). In comparison, under the dark-to-light condition, chlorophyll content remained low and gradually decreased, but increased immediately after exposure to light, which showed the same trend as anthocyanin content (except at $32{ }^{\circ} \mathrm{C}$ ). Thus, both chlorophyll and anthocyanin in the kiwifruit callus accumulate in response to light. In particular, relatively high temperature $\left(32{ }^{\circ} \mathrm{C}\right)$ accelerates the accumulation of chlorophyll but inhibits the accumulation of 
anthocyanin, and vice versa. In brief, temperature might have contrasting effects on anthocyanin and chlorophyll accumulation. However, the interaction between anthocyanin and chlorophyll biosynthesis during fruit ripening requires further investigation.

To evaluate the genetic basis of these phenotypes, the expression levels of five structural genes on the anthocyanin biosynthesis pathway (CHS, CHI, F3' $H, L D O X$, and F3GT), three AccR2R3-MYBs (AcMYB110, AcMYB10, and Acc10227), and HY5 (LONG HYPOCOTYL5, a positive transcription factor that responds to light and is involved in anthocyanin accumulation) [36] were investigated using qRT-PCR. As shown in Figure 4A, after light/dark transition, the expression level of all of these genes noticeably decreased and was then maintained at a relatively low level. After the dark/light transition, the expression levels of all of these genes noticeably increased (and peaked) and then declined slightly but remained at a relatively high level. Thus, the expression of these genes might respond to light. Of note, the expression of $L D O X, F 3 G T$, and MYB10 was higher at $16^{\circ} \mathrm{C}$ compared to $24^{\circ} \mathrm{C}$ and $32{ }^{\circ} \mathrm{C}$, indicating temperature sensitivity. AcF3GT encodes an enzyme dedicated for anthocyanin biosynthesis in the kiwifruit inner pericarp [15]. Among the expression data, that of AcMYB10 was similar to that of AcF3GT, with their expression curves fitting well, Therefore, AcMYB10 might contribute to the accumulation of anthocyanin in the kiwifruit callus by regulating AcF3GT.

The expression levels of genes related to chlorophyll metabolism (CAO, RBCS, GLUTR, CBR, PAO, $P P H$, and $S G R$ ) were also investigated (Figure $4 \mathrm{~B}$ ). The expressions of GLUTR, CBR, and PAO were higher under light conditions compared to dark conditions. The expression of GLUTR was much higher at $32{ }^{\circ} \mathrm{C}$ compared to $24{ }^{\circ} \mathrm{C}$ and $16^{\circ} \mathrm{C}$. Thus, GLUTR expression responds to temperature, promoting chlorophyll biosynthesis in the kiwifruit callus. However, the other six genes showed no similar expression patterns corresponding to chlorophyll content. Thus, a more complicated mechanism, other than light and temperature, likely regulates the green pigmentation of kiwifruit.

\subsection{Sequence Analysis, Transcriptional Activation Ability, and Expression Characteristic of Acc00493 (AcMYB10)}

From above, Acc00493 showed a close phylogenetic relationship with the R2R3-MYBs responsible for anthocyanin biosynthesis in Arabidopsis. In addition, it was strongly expressed in the inner pericarp of red-fleshed kiwifruit, and its expression level was correlated with light-/temperature-regulated pigmentation in the kiwifruit callus. Thus, Acc00493 was screened as a candidate gene involved in the biosynthesis of anthocyanin in kiwifruit. To investigate the de novo function of Acc00493, we cloned the full-length CDS of Acc00493 from 'Hongyang' genomic DNA. The coding sequence of Acc00493 is identical to AcMYB10 (Genbank: MG581953.1), but has one nucleotide difference with AcMYB75 (Genbank: KX349735.1) (Figure 5A), suggesting both AcMYB10 and AcMYB75 should probably be designated to locus Acc00493. Since this SNP (single nucleotide polymorphism) will cause a change in one amino acid of the protein sequence, we further amplificated the full-length DNA of Acc00493 in 'Hongyang'. No AcMYB75 was detected using either PCR products or TA clones (Supplementary Figure S5), indicating only one allele, $A c M Y B 10$, is at this locus in 'Hongyang' (Supplementary Figure S1B). Because $A c M Y B 75$ was identified using an RNA-seq dataset, and no DNA sequence and flanking sequence of $A c M Y B 75$ have been reported, we speculate that $A c M Y B 75$ is probably a different allele of Acc00493 in another cultivar, but not 'Hongyang', or a paralog of Acc00493, which had not been assembled in the present two A. chinensis genomes, or just a sequencing or assembly error. Thus, we designated Acc00493 as AcMYB10 from here on. 


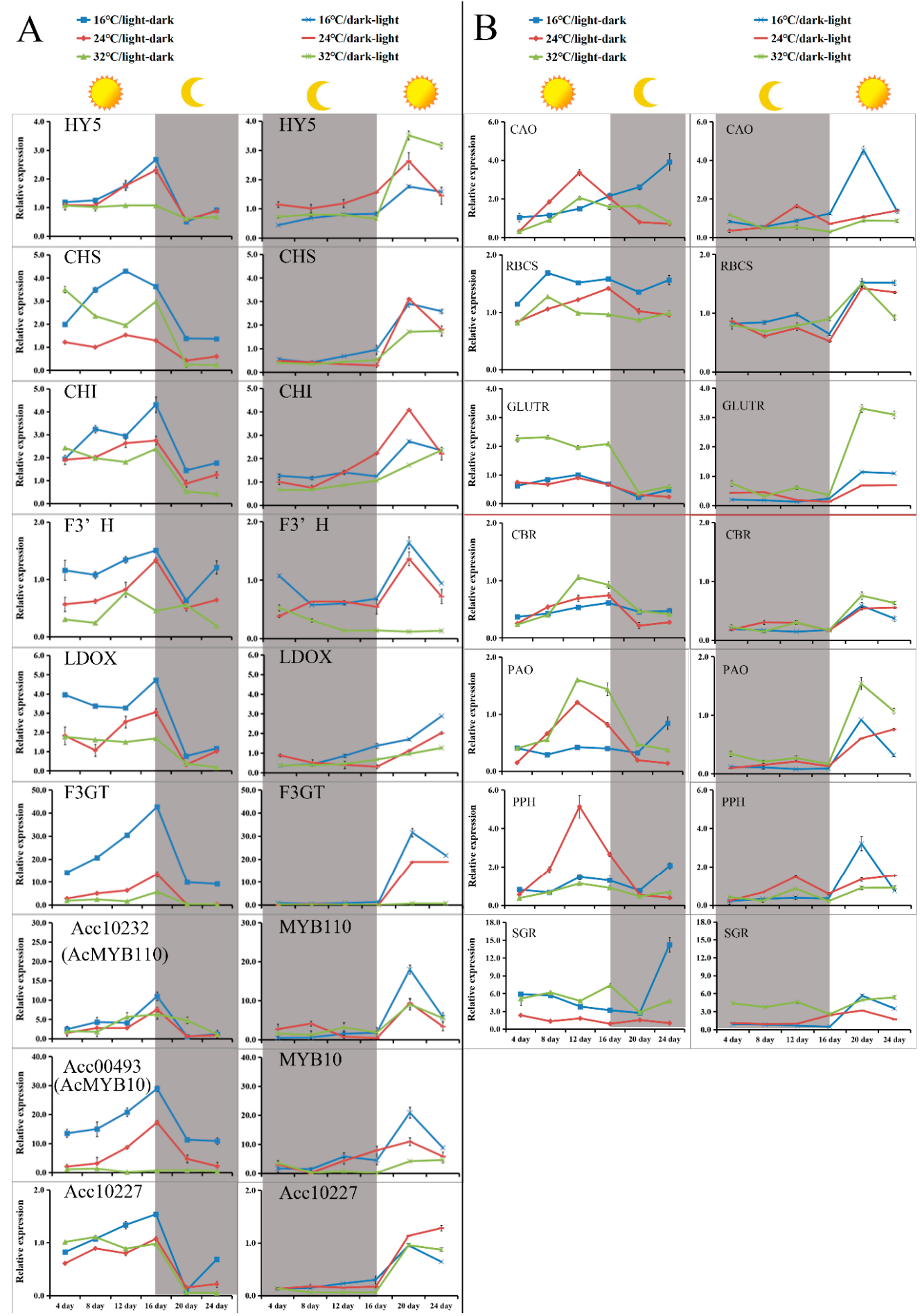

Figure 4. Relative expression levels of anthocyanin- and chlorophyll-related genes in the callus over 24 days under different culture conditions. (A) Relative expression levels of the structural genes and AccR2R3-MYBs in the anthocyanin biosynthesis pathway. HY5, LONG HYPOCOTYL5; CHS, Chalcone synthase; $\mathrm{CHI}$, chalcone isomerase, $F 3^{\prime} \mathrm{H}$, flavanone $3^{\prime}$-hydroxylase; $L D O X$, leucoanthocyanidin dioxygenase; F3GT, UDP flavanone- 3-Oglucosyltransferase. (B) Expression levels of genes related to chlorophyll metabolism. CAO, Chlorophyll a oxygenase; RBCS, Small subunit of ribulose-1,5-bisphosphate Carboxylase; GLUTR, Glutamyl tRNA reductase; $C B R$, Chlorophyll b reductase; $P A O$, Pheophorbide a oxygenase; $P P H$, Pheophytin pheophorbide hydrolase; SGR, Stay-green. Results represent the mean $\pm \mathrm{SD}$ of three replicates. 

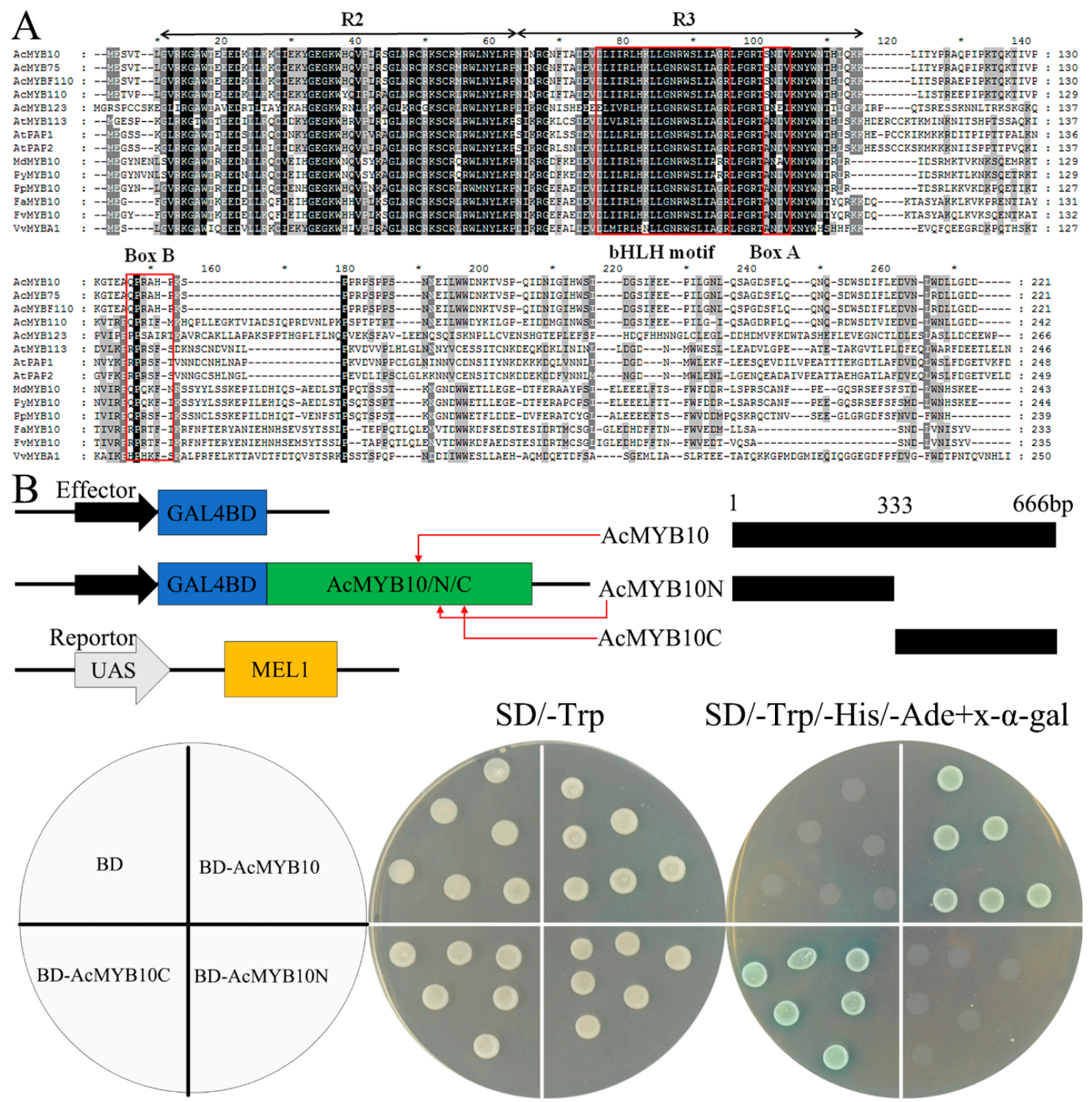

Figure 5. Analyses of the AcMYB10 activation domain. (A) Amino acid sequence alignment of Acc00493 (AcMYB10) and other plant anthocyanin-promoting MYBs. Totally conserved residue is highlighted in black and partial conserved is highlighted in gray. R2 and R3 MYB motifs are indicated by arrows. The bHLH motif indicates the residues needed for the interaction with the bHLH partner; box A and box B are well-conserved in anthocyanin-promoting MYBs. The asterisks indicate the number of amino acid. The GenBank accession numbers are: AcMYB75 (APZ74276.1), AcMYBF110 (AYJ72555.1), AcMYB110 (AHY00342.1), AcMYB123 (QAT77713.1), AtMYB113 (NP_176811.1), AtPAP1 (Q9FE25.1), AtPAP2 (Q9ZTC3.1), MdMYB10 (ABB84753.1), PyMYB10 (ADN26574.1), PpMYB10 (ADK73605.1), FaMYB10 (ABX79947.1), FvMYB10 (ABX79948.1), VvMYBA1 (AGH68552.1). (B) Transcriptional activity assay of AcMYB10. Schematic of the full length and three truncated fragments (AcMYB10N and AcMYB10C) of $A c M Y B 10$ used for constructing vectors. Effectors and reporter used for transcriptional activation activity assay. Growth of yeast cells (strain AH109) transformed with each of the three vectors or pGBKT7 empty vector (used as a negative control) on SD/-Trp or SD/-Trp/-His/-Ade, with the addition of X- $\alpha$-gal. The layout of the effectors is shown in the pie chart on the left.

The AcMYB10 protein contained a highly conserved N-terminal R2R3 repeat of a DNA-binding domain with a bHLH motif which was functionally important for the interaction between MYB and R/B-like bHLH proteins [37] (Figure 5A). To determine the transcriptional activation activity of AcMYB10, 
the full-length and two truncated CDS of AcMYB10 (AcMYB10N[1-111aa] and AcMYB10C[112-222aa]) were fused to pGBKT7 to generate three effectors. The effectors were separately transferred to the yeast containing a MEL1 reporter (Figure 5B). All of the yeast cells grew well on the SD medium lacking tryptophan (SD/-Trp), whereas only yeast cells transformed with the effectors containing BD-AcMYB10 or BD-AcMYB10C grew and displayed GAL4 activity on the medium supplemented with X- $\alpha$-gal (Figure 5B). These results demonstrate that the transcriptional activation activity of AcMYB10 and the AcMYB10C region is necessary for transactivation. Thus, the C-terminal region within AcMYB10 may contain key residues that were important for transactivation efficiency, as previously reported [38].

To investigate the expression pattern of $A C M Y B 10$ during the fruit development of red-fleshed kiwifruit, four stages of the red-fleshed A. chinensis cv. Hongyang and yellow-fleshed A. chinensis cv. Jinyan fruits were sampled for expression and anthocyanin analysis. As shown in Supplementary Figure S6, low anthocyanin levels and low expressions of AcLDOX, AcF3GT, and AcMYB10 were detected in the inner pericarp of 'Jinyan' during fruit development. In comparison, an increasing anthocyanin content and expression of AcMYB10, AcF3GT, and AcLDOX were detected in the inner pericarp of 'Hongyang' along with fruit development and pigmentation. These results support that anthocyanin accumulation in the inner pericarp is associated with the coordinated action of AcMYB10, $A c F 3 G T$, and AcLDOX during fruit development and ripening, supporting previous work [17].

2.7. Gene Functional Study by Overexpression of AcMYB10 in A. chinensis cv. Hongyang and Using the VIGS (Virus-Induced Gene Silencing) System in A. chinensis cv. Hongshi No. 2

To further reveal the function of AcMYB10, the recombinant vector 35S:AcMYB10 was transformed to the 'Hongyang' callus for generating transgenic kiwifruit plants. The leaves of 35S:AcMYB10 (OE) had a clear red color at the young stage, whereas the wild type (WT) showed no obvious abnormal phenotype (Figure 6A). The anthocyanin content of OE lines was significantly higher than that of WT, and the expressions of $A c M Y B 10, A c F 3 G T$, and AcLDOX were also significantly higher in OE compared to WT (Figure 6B,C).

Anthocyanins belong to the flavonoid class of compounds. The accumulation of anthocyanins was associated with multiple upstream flavonoid metabolites [20]. Thus, to investigate metabolite dynamics caused by the over-expression of AcMYB10 comprehensively, a widely targeted metabolomics method was employed to identify and quantify relative flavonoid metabolites in the leaf of transgenic 'Hongyang' (OE) [39]. A total of 224 flavonoids were identified in OE and WT (for details, see Supplementary Table S3). A total of 47 differential metabolites had significantly different contents between OE and WT, including 13 anthocyanins, 2 polyphenols, 1 flavonoid, 5 flavanones, 22 flavones, 1 flavonol, 1 proanthocyanidin, and 2 isoflavones (Figure 6D). Twenty-seven and 20 of these metabolites were up-regulated and down-regulated in OE, respectively (Supplementary Table S3). Of note, all seven identified cyanidin-, two peonidin-, and one pelargonidin-based anthocyanins were up-regulated in $\mathrm{OE}$, whereas two peonidin- and two pelargonidin-based anthocyanins were down-regulated. Because cyanidins are the main anthocyanin in A. chinensis responsible for the red color [12], these results indicate that the overexpression of AcMYB10 promotes the biosynthesis of metabolites in the cyanidin pathway, leading to the red color of transgenic young leaves of OE.

This study used VIGS on 'Hongshi No. 2' fruit to suppress AcMYB10. After 7 days, the expression of AcMYB10 was strongly suppressed in fruit infiltrated with TRV1and TRV2:AcMYB10, leading to delayed pigmentation in infiltrated fruit. In comparison, pigmentation was normal in the fruits infiltrated with TRV1 and TRV2 (Figure 7). In addition, the anthocyanin content was much lower for the fruit treated with TRV1 and TRV2:AcMYB10 compared to fruit treated with TRV1 and TRV2 (Figure 7). Overall, these results demonstrate that $A c M Y B 10$ is involved in anthocyanin accumulation in red-fleshed kiwifruit. 
A
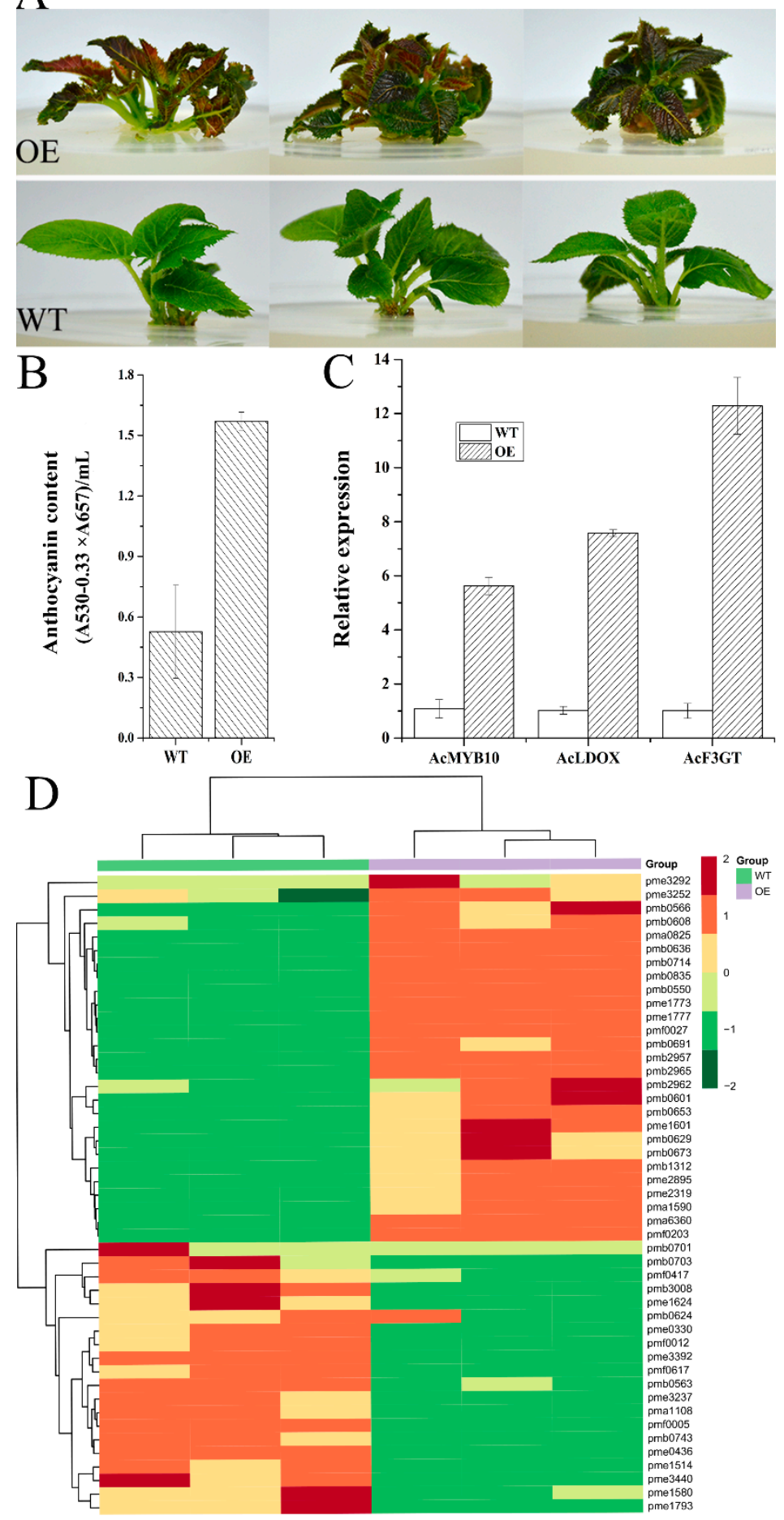

Figure 6. Wild type (WT) and transgenic 'Hongyang' (OE) expressing AcMYB10 under the control of the 35 S promoter. (A) Phenotypes of transgenic and wild-type 'Hongyang' kiwifruit. (B) Anthocyanin content in transgenic kiwifruit leaves. (C) Expression level of AcMYB10, AcLDOX, AcF3GT in wild-type and transgenic kiwifruit leaves. (D) Heat map visualization of flavonoid metabolites. Each metabolite is represented by a single row. Dark red indicates relatively high abundance, whereas metabolites with relatively low abundance are shown in dark green (color key scale on the right of the heat map). 
A

A
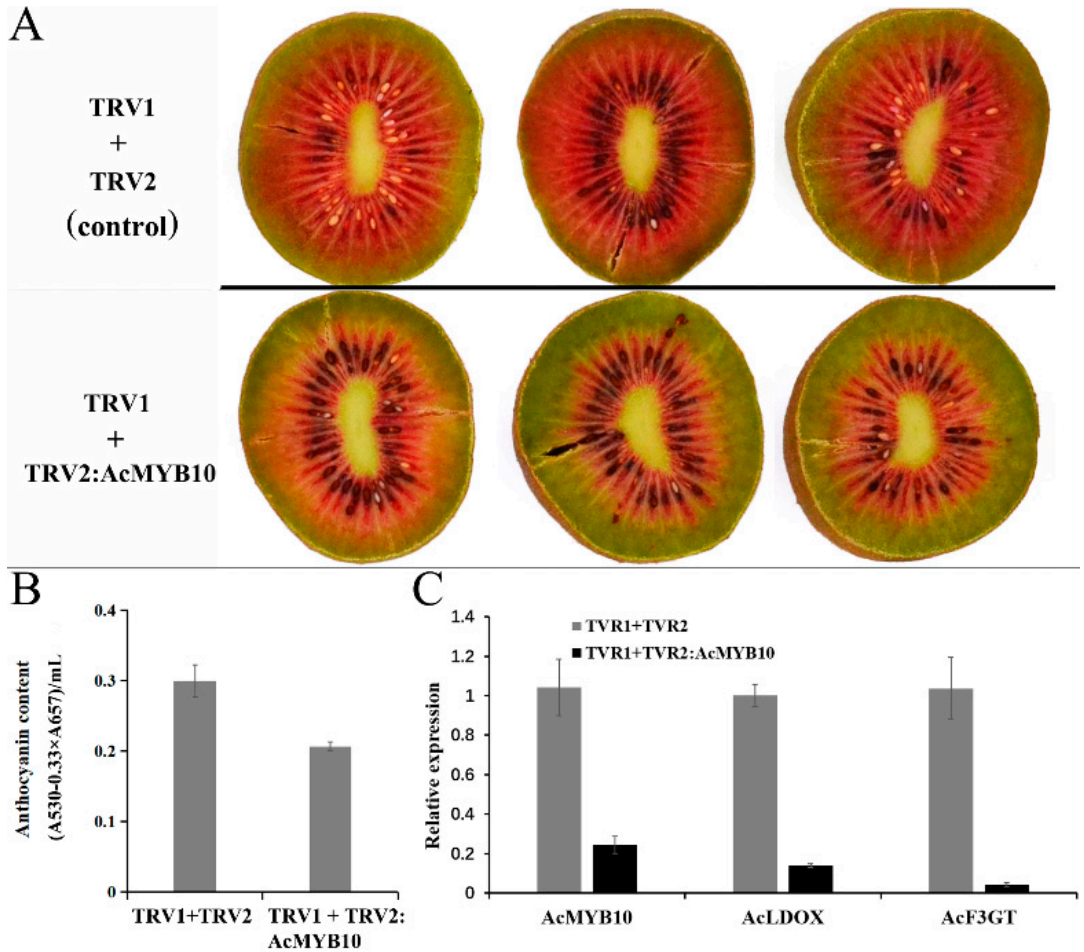

Figure 7. Functional analysis of $A c M Y B 10$ using virus-induced gene silencing (VIGS). (A) Silencing of $A c M Y B 10$ in 'Hongshi No. 2' fruits. The photograph was taken seven days after infiltration. (B) Anthocyanin content of treated fruits. (C) Expression level of AcMYB10, AcLDOX and AcF3GT in the inner pericarp of treated fruits.

\subsection{Subcellular Localization Analysis}

Because MYBs have been widely reported to interact with bHLH, leading to the activation of structural genes on anthocyanin biosynthesis [40], we investigated the subcellular distribution of the AcMYB10 protein and AcBHLH42. The fusion vector (AcMYB10-YFP and AcBHLH42-YFP) and the control vector (YFP) were transiently expressed in tobacco leaves, respectively. Confocal imaging of the epidermis showed that just YFP was detected throughout the entire cell, while AcMYB10-YFP and AcBHLH42-YFP fusion proteins were exclusively localized in the nucleus (Supplementary Figure S7). Localization in the nucleus was confirmed using the co-transformation of a nucleus marker gene fused to mCherry in the epidermis. This result indicates that both AcMYB10 and AcBHLH42 are nuclear proteins. Thus, physical interaction between them is possible.

\subsection{Protein-Protein Interactions between AcMYB10 and AcbHLH42}

The yeast two-hybrid assay was employed to demonstrate the interaction between AcMYB10 and AcBHLH42 proteins. Yeast harboring AcBHLH42-AD + AcMYB10-BD and AcBHLH42-BD + $A c M Y B 10-A D$ grew well on the quadruple-selection medium. In comparison, the negative control that contained BD + AcMYB10-AD, BD + AcBHLH42-AD, AD + AcBHLH42-BD, and AD + AcMYB10-AD did not grow (Figure 8). Thus, a physical interaction occurs between AcMYB10 and AcbHLH42 proteins in vitro. Subsequently, two bait vectors encoding different $A c M Y B 10$ regions [AcMYB10N(1-111aa) and AcMYB10C (112-222aa)] fused to the BD and co-transformed to the yeast strain AH109, along with the prey vector $A c B H L H 42-\mathrm{AD}$, to identify the interaction region. The yeast colonies expressing AcBHLH42 and N-terminal AcMYB10 truncations (AcMYB10N) grew well on the medium (SD/-Ade/-His/-Leu/-Trp) containing $15 \mathrm{mM}$ 3-amino-1,2,4-triazole (3AT). In comparison, yeast colonies expressing AcBHLH42 and AcMYB10 constructs containing the C-terminal region (AcMYB10C) did not grow on the medium (SD/-Ade/-His/-Leu/-Trp) containing 15 mM 3AT. Thus, the N-terminal 
was essential for protein-protein interactions between AcMYB10 and AcBHLH42 (Figure 8). Therefore, the bHLH motif (located on the N-terminal) is likely a key DNA-binding domain for the interaction between $A c B H L H 42$ and $A c M Y B 10$, as previously reported [37]. These results suggest AcMYB10 can interact with $A c B H L H 42$ and may further promote anthocyanin accumulation in kiwifruit.

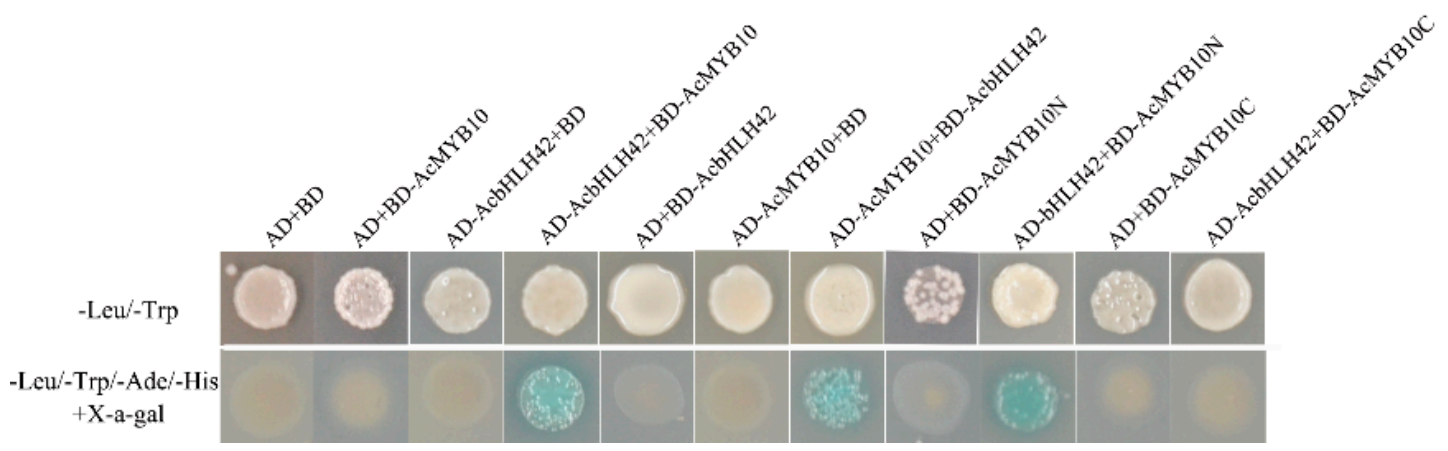

Figure 8. Interactions between $A c M Y B 10$ and $A c b H L H 42$ detected by the yeast two-hybrid assay. AH109 yeast cells containing plasmids were grown on double-and quadruple-selection media. The $\mathrm{X}-\alpha$-gal assay was performed to confirm positive interactions.

\subsection{AcMYB10 and AcbHLH42 Regulate the Promoter Activities of AcLDOX and AcF3GT}

To evaluate the ability of $A c M Y B 10$ and AcbHLH42 to activate to the promoter sequences of the anthocyanin biosynthetic genes $A c L D O X$ and AcF3GT transcriptionally, a dual luciferase system was used on transiently transfected N. benthamiana leaves. As shown in Figure 9A, AcMYB10 activated the promoters of AcLDOX ( $\sim 38$ fold compared to the control) and AcF3GT ( 15 fold). Sequence analysis showed that both AcLDOX and AcF3GT promoters contain three 7-bp MYB recognizing elements (MREs) (Supplementary Figure S8), which might be the target of MYBs [41]. As shown in Figure 9A, a set of deletion mutation constructs was generated to test the functional roles of these MREs. As expected, each MRE deletion on AcLDOX and AcF3GT promoters partially reduced the transactivation of AcLDOX and AcF3GT caused by AcMYB10. The deletion of fragments that did not contain MRE also reduced transactivation; thus, other recognition elements or motifs might also be important for transactivation caused by AcMYB10. In addition, the transcriptional activation ability of AcF3GT/AcLDOX + 35S:AcMYB10 + 35S:AcbHLH42 was much higher than that of AcF3GT/AcLDOX + 35S:AcMYB10 or AcF3GT/AcLDOX + 35S:AcbHLH42 (Figure 9B). Therefore, AcMYB10 and AcbHLH42 have a synergistic function in activating the promoters of AcF3GT and AcLDOX.

\subsection{AcMYB10 Interacts with AcbHLH42 to Activate Anthocyanin Biosynthesis in N. tabacum Leafs and A. arguta Fruit}

To verify the function of $A c M Y B 10$ and $A c b H L H 42$ in anthocyanin biosynthesis, their transient expression was examined in the tobacco leaf and A. arguta fruit. A. tumefaciens strain GV3101 harboring the recombinant plasmids of 35S:AcMYB10 and 35S:AcbHLH42 were syringe-infiltrated to the abaxial surfaces of expanding $N$. tabacum leaves. Red pigmentation was observed at the injection sites of 35S:AcMYB10 and co-injected 35S:AcMYB10 and 35S:AcbHLH42 six days after injection (Figure 10A). In comparison, the injection sites remained green in leaves injected with empty vector or 35S:AcBHLH42. Anthocyanin content was detected in 35S:AcMYB10 and co-injected 35S:AcMYB10 + 35S:AcBHLH42, but not in the empty vector and 35S:AcBHLH42. The anthocyanin content in co-expressing 35S:AcMYB10 and 35S:AcbHLH42 was significantly higher than that in 35S:AcMYB10. The five structural genes (NtCHS, NtF3H, NtDFR, NtANS, and NtUFGT) in the anthocyanin biosynthesis pathway showed highest expression in co-injected 35S:AcMYB10 and 35S:AcbHLH42 (Figure 10A). 
A
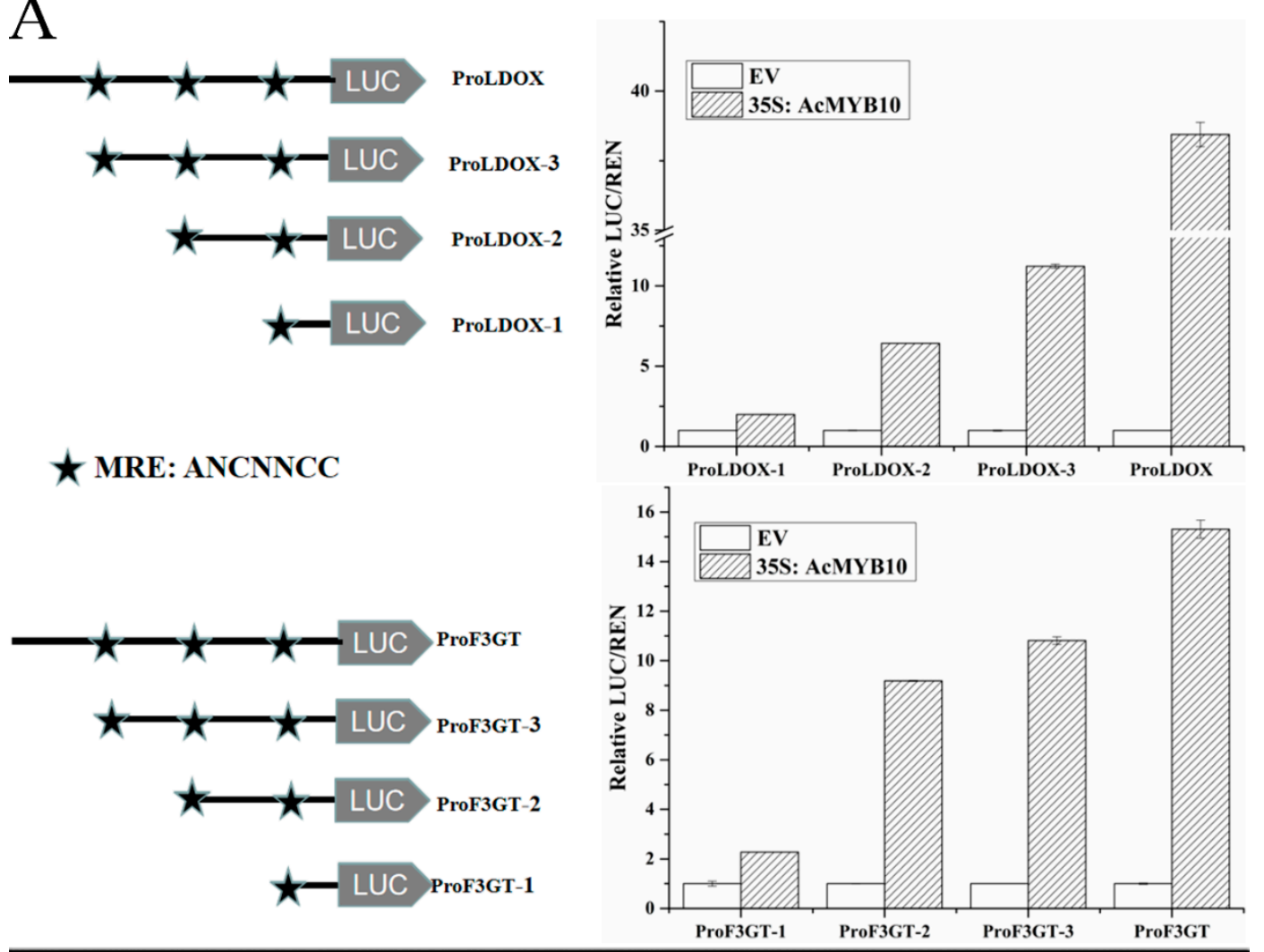

B
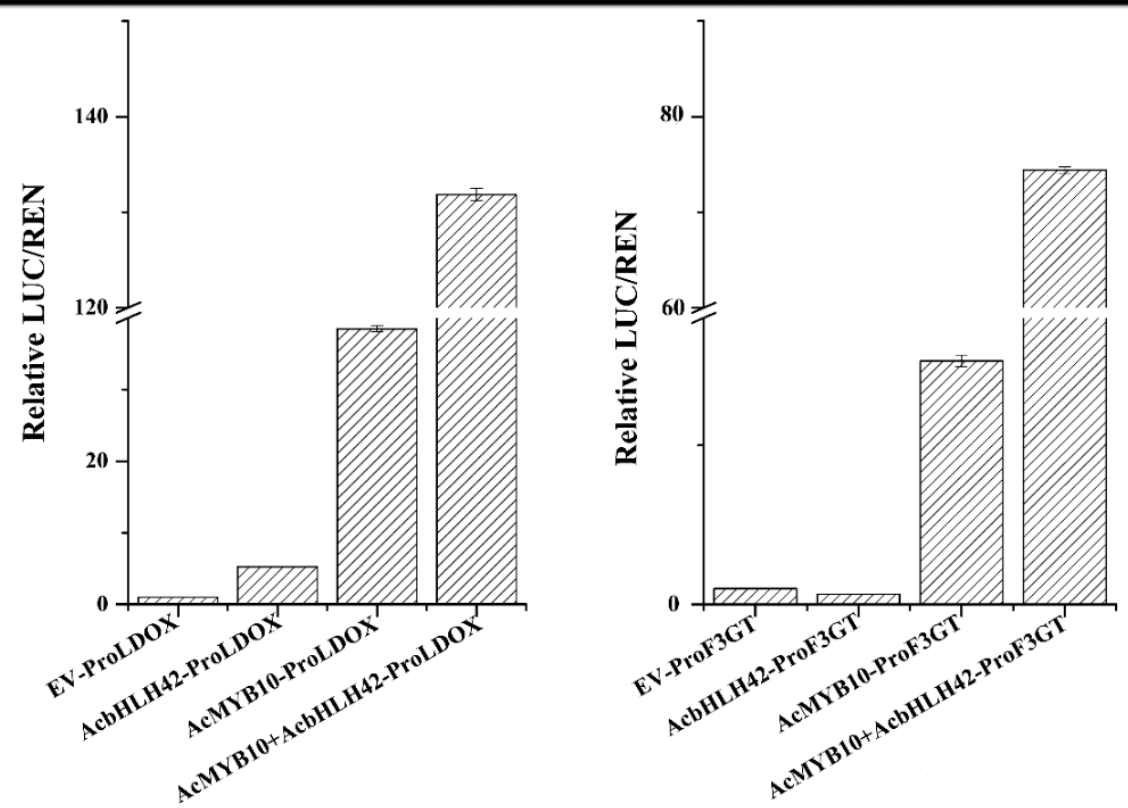

Figure 9. Transcriptional activation analysis of AcMYB10-mediated and AcBHLH42-mediated induction of AcLDOX and AcF3GT promoter activities. (A) Diagrams showing various DNA fragments of the two selected promoters linked to the firefly luciferase reporter. (B) AcMYB10-mediated and AcBHLH42-mediated induction of AcLDOX and AcF3GT promoter activities. LUC, Firefly luciferase activity; REN, Renilla luciferase activity. The ratio of LUC/REN of the empty vector (EV) plus promoter was used as a calibrator (set as 1 ). Results represent the mean \pm SD of six replicates. 

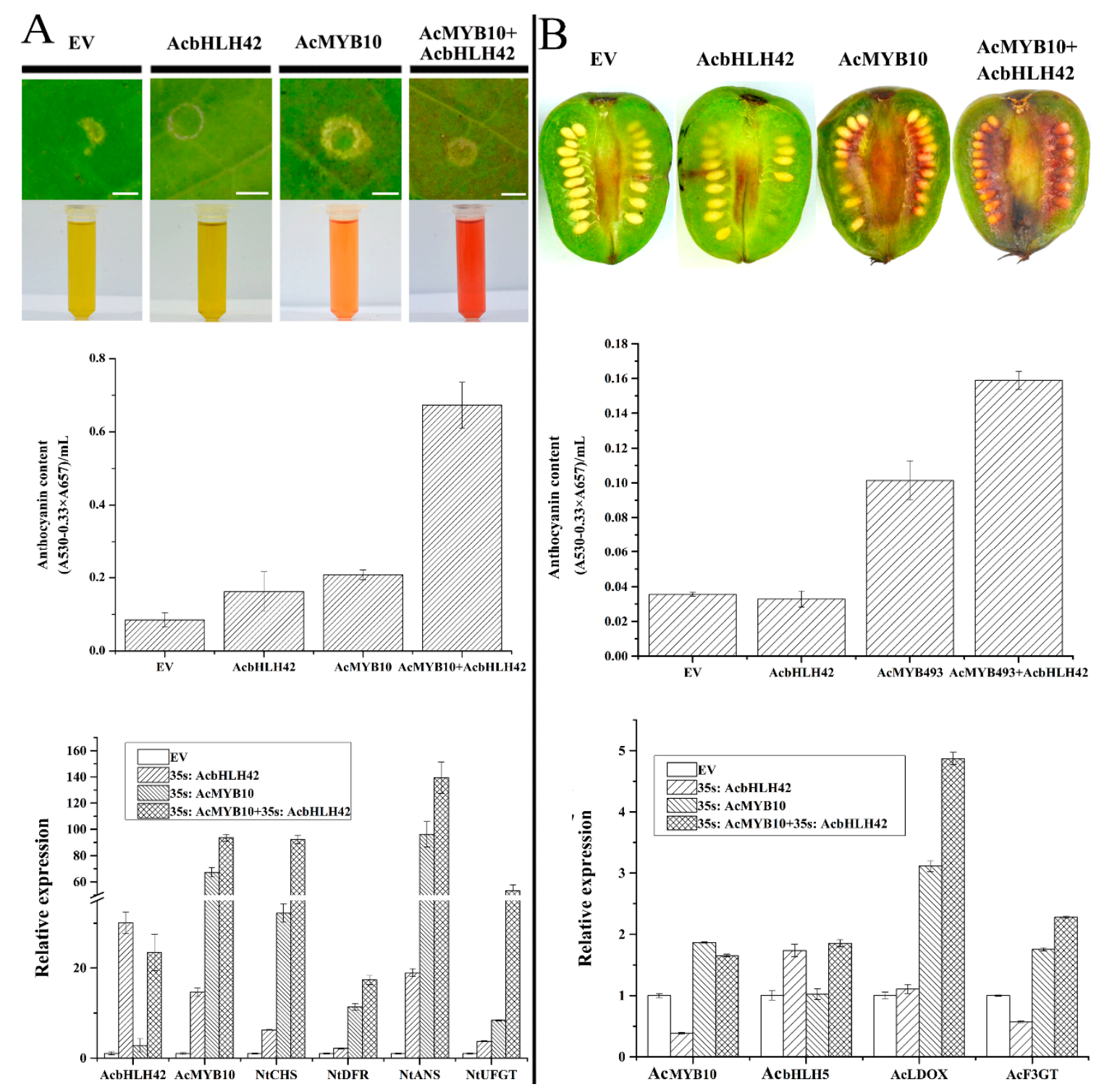

Figure 10. The combinatorial action of $A c M Y B 10$ and AcbHLH42 determines anthocyanin production in transiently expressed $N$. tabacum leaves and A. arguta. Color changes induced by transiently expressing either 35S:AcMYB10 or 35S:AcbHLH42 alone, or co-expressing 35S:AcMYB10 and 35S:AcbHLH42 in (A) N. tabacum leaves and (B) A. arguta fruit. The liquid in test tubes are fresh extracts from the individual infiltrated patches. The bar scales are $4 \mathrm{~mm}$. Total anthocyanin content measured in (A) $N$. tabacum leaves and (B) A. arguta fruit. Anthocyanin content was determined 6 days after infiltration. Expression analysis of AcMYB10, AcBHLH42, and key anthocyanin biosynthesis genes in (A) N. tabacum leaves and (B) A. arguta fruit. Results represent the mean \pm SD of three replicates.

To demonstrate the function of $A c M Y B 10$ and AcbHLH42 in the fruit tissue of kiwifruit, the same vectors were injected into the fruit of $A$. arguta cv. Baobeihong (Figure 10B). The highest levels of anthocyanins were detected in the fruits co-overexpressing AcMYB10 and AcbHLH42, which corresponded to the increased expression levels of AcMYB10, AcbHLH42, AcF3GT, and AcLDOX. Thus, AcMYB10 might accelerate anthocyanin biosynthesis through interacting with AcbHLH42 in the fruit tissue of kiwifruit. 


\section{Materials and Methods}

\subsection{Plant Materials, Treatment, and Nucleic Acid Extraction}

The kiwifruit callus was induced from Actinidia chinensis cv. Hongyang as previously described [42,43]. In the light and temperature treatment experiment, the callus was incubated under the following conditions: $16{ }^{\circ} \mathrm{C} /$ light (intensity of $80 \mu \mathrm{mol} \mathrm{m}^{-2} \mathrm{~s}^{-1}$ ), $24^{\circ} \mathrm{C} /$ light, $32{ }^{\circ} \mathrm{C} /$ light, $16{ }^{\circ} \mathrm{C} /$ dark, $24^{\circ} \mathrm{C} /$ dark, and $32^{\circ} \mathrm{C}$ /dark. The callus was sampled at $0,4,8,12,16,20$, and 24 days after treatment.

In the VIGS experiment, Agrobacterium was injected into the fruit of 'Hongshi No. 2'. In the transient expression experiment, Agrobacterium was injected into the fruit of A. arguta cv. Baobeihong, a purple $A$. arguta var. purpurea cultivar. The samples of 'Hongshi No. 2' and 'Baobeihong' were collected from a germplasm garden at Wufeng mountain, Enshi city, Hubei province.

The genomic DNA used in this study was extracted from 'Hongyang' leaves using a DNA extraction kit (DN38, Aidlab, Beijing). Total RNA was extracted using HiPure Plant RNA kits (R4151-03, Magen, Shanghai). The cDNA libraries were constructed by using HiScript II QRT SuperMix (R223-01, Vazyme, Nanjing).

\subsection{Genome-Wide Analysis and Expression Profile of Kiwifruit R2R3-MYB Genes}

The hidden Markov model (HMM) profile for the MYB binding domain (PF00249) was downloaded from Pfam (http://pfam.sanger.ac.uk/) and subsequently exploited to identify MYB genes from the 'Red5' kiwifruit genome using HMMER 3.2. The default parameters were adopted, and the cutoff value was set to 0.01 . To confirm the core MYB domains, putative MYB sequences were further examined using a conserved domain search (https://www.ncbi.nlm.nih.gov/Structure/cdd/wrpsb.cgi) and InterProScan (http://www.ebi.ac.uk/interpro/). All of the raw R2R3-MYB proteins were manually inspected to generate a final MYB dataset. Subsequently, according to the identified gene IDs, the transcript sequences, coding sequences (CDS), genome sequences, chromosome locations, and annotations of these MYB superfamily proteins were extracted from the 'Red5 genome' [44] using Perl script. MEME Suite 5.0.5 [43] was used to identify motifs in the 155 MYB protein sequences with the following parameters: the maximum number of motifs was set to 20 , and the optimum width of each motif was set from 10 to 100 residues. The chromosome location and the exon/intron structures of kiwifruit R2R3-MYB genes were plotted using the R script. Phylogenetic trees were constructed based on the conserved domain of R2R3-MYB proteins, with a bootstrap analysis of 1000 replicates using MEGA 7 software [45]. The sequences and annotation of 126 Arabidopsis R2R3-MYB proteins were downloaded from the TAIR (The Arabidopsis Information Resource: http://www.arabidopsis.org/). The upstream 2 $\mathrm{kb}$ genomic DNA sequences of anthocyanin-related AccR2R3-MYBs (labelled with a dotted line) were submitted to the Plant CARE database (http://bioinformatics.psb.ugent.be/webtools/plantcare/html/) to identify the cis-elements.

The callus, leaf, flower, and inner pericarp of 'Hongyang' were sampled for the RNA-seq. The transcript abundance of kiwifruit R2R3-MYB genes was calculated as fragments per kilobase of the exon model per million mapped reads (FPKM). The raw FPKM data were normalized to log2(FPKM+1) for downstream analysis. The hierarchical clustering of the expression data was constructed in the $R$ package.

\subsection{Quantitative Real-Time RT-PCR}

RT-PCR was performed on Quant Studio 6 Flex (Life Technologies Corporation, Carlsbad, CA, USA), following the manufacturer's instructions for AceQ qPCR SYBR Green Master Mix Q131-02 (Vazyme, Nangjing, China). The reactions were carried out as follows: $95^{\circ} \mathrm{C}$ for $30 \mathrm{~s}$, followed by 40 cycles at $95{ }^{\circ} \mathrm{C}$ for $10 \mathrm{~s}, 60^{\circ} \mathrm{C}$ for $30 \mathrm{~s}$, and $72{ }^{\circ} \mathrm{C}$ for $20 \mathrm{~s}$. Melt-curve analyses were carried out as follows: $15 \mathrm{~s}$ at $95^{\circ} \mathrm{C}, 1 \mathrm{~min}$ at $60^{\circ} \mathrm{C}, 30 \mathrm{~s}$ at $95^{\circ} \mathrm{C}$, and $15 \mathrm{~s}$ at $60^{\circ} \mathrm{C}$. The $2^{-\Delta \Delta \mathrm{Ct}}$ method was employed with Achn107181 (Actin) as an endogenous control [46]. All of the primers used in this study are shown in Supplementary Table S4. 


\subsection{Determining the Concentration of Anthocyanin, Chlorophyll, and Flavonoid Metabolites}

Total anthocyanin content was extracted and determined according to the methods described by Shin et al. [47] and Lim et al. [48]. Briefly, 0.1 g powdered tissue samples were incubated in $600 \mu \mathrm{L}$ extraction buffer (methanol containing $1 \% \mathrm{HCl}$ ) for $6 \mathrm{~h}$ at $4{ }^{\circ} \mathrm{C}$ with moderate shaking. After extraction, $200 \mu \mathrm{L}$ of water and $200 \mu \mathrm{L}$ of chloroform were added to each sample, and absorbances were read at $530 \mathrm{~nm}$ and $657 \mathrm{~nm}$ using a microplate reader. Anthocyanin content was determined using the following equation: A530 $-0.33 \times \mathrm{A} 657$. Chlorophyll $a$ and $b$ were extracted and determined following a method described by Hiscox and Israelstam [49]. Briefly, $0.1 \mathrm{~g}$ powered sample was extracted with $5 \mathrm{~mL}$ dimethylsulfoxide for $72 \mathrm{~h}$ with shaking in the dark. Absorbances of $2 \mathrm{~mL}$ of the sample extracts were read at $663 \mathrm{~nm}$ and $645 \mathrm{~nm}$ using a spectrophotometer. Chlorophyll content was determined using the following equation: $\mathrm{Chl} a=(12.7 \times$ OD $663-2.69 \times$ OD645) $\times 5 / 0.1 \times 1000 \times 2$; Chl $b=$ $(22.9 \times$ OD $645-4.68 \times$ OD663 $) \times 5 / 0.1 \times 1000 \times 2$. Flavonoid metabolites were extracted, isolated, and identified according to a method described by Wang et al. [50]. Briefly, $100 \mathrm{mg}$ powdered sample was extracted overnight at $4{ }^{\circ} \mathrm{C}$ with $1.0 \mathrm{~mL} 70 \%$ aqueous methanol. Following centrifugation $(10,000 \times g$ for $10 \mathrm{~min}$ ), the extracts were passed through the SPE cartridge and filtrated before LC-MS/MS analysis. The sample extracts were analyzed using a liquid chromatography-electrospray ionization tandem mass spectrometry (LC-ESI-MS/MS) system (HPLC, UFLC SHIMADZU CBM30A system, www.shimadzu.com.cn/; MS, Applied Biosystems 4500 QTrap, www.appliedbiosystems.com.cn/). The corresponding relative metabolite contents were represented as chromatographic peak area integrals.

\subsection{Isolation and Sequence Alignments and Transcriptional Activation Analysis of AcMYB10}

Full-length DNA sequences of Acc00493 were amplificated from 'Hongyang' genomic DNA. PCR products were cloned into pEASY-Blunt Cloning Vector, and then the recombinant plasmid was transformed into Escherichia coli (trans-T1) cells using pEASY-Blunt Cloning Kit CB101 (Transgen, Beijing, China). Ten amplified PCR products and ten monoclonals were sequenced by Sanger sequencing. Full-length CDS of AcMYB10 (Acc00493) and AcBHLH42 (Acc19563) were amplified from the cDNA library of the inner pericarp of 'Hongyang'. The full length and two truncated parts of AcMYB10 (AcMYB10N: 1-111aa or AcMYB10C: 112-222aa) CDS were amplified and cloned to the pGBKT7 vector (pGBKT7-AcMYB10/AcMYB10N/AcMYB10C). The fusion vectors and empty vector (pGBKT7) were separately transformed to the yeast (Saccharomyces cerevisiae) strain AH109, harboring a MEL1 reporter. Transcriptional activation activity of the transformed yeast cells was determined after incubation at $30{ }^{\circ} \mathrm{C}$ for 3 days on SD/-Trp or SD/-Trp/-His/-Ade medium supplemented with $0.02 \mathrm{mg} / \mathrm{mL}$ X- $\alpha$-gal CX11922 (Coolaber, Beijing, China).

\subsection{Dual Luciferase Assay of Transiently Transformed Tobacco Leaves}

The putative promoters of AcF3GT (Acc20131) (approximately $\sim 1 \mathrm{~kb}$ upstream of the ATG), AcLDOX (Acc28876) (approximately $\sim 1.5 \mathrm{~kb}$ upstream), and various deletions of the promoter fragments containing MRE cis-elements were PCR-amplified from 'Hongyang' genomic DNA. All of these fragments were inserted to the cloning site of pGreen0800-LUC using In-Fusion cloning CU201-02 (TransGen, Beijing, China), and were then transformed to A. tumefaciens GV3101. The CDSs of AcMYB10 and AcBHLH42 were respectively cloned to the pCAMBIA1301 over-expression vector driven by the $35 S$ promoter using In-Fusion cloning CU201-02. Agrobacterium infiltration and measurement of luciferase activity were conducted as described previously [51,52].

3.7. Gene Functional Study Using the VIGS System in Kiwifruit and Over-Expression of AcMYB10 in A. chinensis, A. arguta, and N. tabacum

The 400-666 fragment of AcMYB10 was amplified and inserted to pTRV2 to generate TRV2:AcMYB10 vector. A. tumefaciens strain GV3101 containing TRV1 + TRV2:AcMYB10 or TRV1 + TRV2 was injected to the fruit of 'Hongshi No. 2' at about 100 days after pollination (DAP) from 
the fruit lateral surface at four opposite points around the central section. The detailed experimental procedure is presented in [53].

Fresh calluses of 'Hongyang' were inoculated with A. tumefaciens strain EHA105 containing the 35S:AcMYB10. A high-efficiency transformation procedure for kiwifruit was performed, as previously reported [54]. The regenerated calluses were selected on medium containing $75 \mathrm{mg} / \mathrm{L}$ of $\mathrm{G} 418$ and transferred to fresh selection medium for bud induction under 12:12 (light: dark) conditions at $26{ }^{\circ} \mathrm{C}$.

The A. tumefaciens GV3101 strains containing 35S:AcMYB10, 35S:AcBHLH42, 35S:AcMYB10 + 35S:AcBHLH42, and the empty vector were injected into the abaxial leaf surface of six-week-old $N$. tabacum and 50 DAP fruits of $A$. arguta cv. Baobeihong, respectively. The phenotype was observed and sampled 6 days after injection.

\subsection{Subcellular Localization Analysis}

The CDSs of AcMYB10 and AcbHLH42 (without the stop codon) were cloned to the 101YFP (yellow fluorescent protein) vector under the control of the CaMV35S promoter. The fusion construct (AcMYB10-YFP and AcBHLH42-YFP) or the control vector (YFP), as well as cell nucleus marker VirD2NLS fused to mCherry, was transformed to A. tumefaciens strain GV3101 and co-infiltrated to the leaves of $N$. benthamiana with the suspension, as previously described [55]. After $48 \mathrm{~h}$ incubation at $25^{\circ} \mathrm{C}$, tobacco leaves were used for YFP and RFP fluorescence signal observations using a laser-scanning confocal microscope TCS-SP8 (Leica-Microsystems, Wetzlar, Germany).

\subsection{Yeast Two-Hybrid (Y2H) Assays}

The full lengths of AcMYB10 and AcbHLH42 CDS were respectively inserted to the pGADT7 vector, which were designated as "AD-AcMYB10" and "AD-AcMYB10" constructs in the present study. To generate binding domain (BD) constructs, the following fragments were amplified: full-length AcMYB10, N-terminal region (AcMYB10N:1-111aa), C-terminal region (AcMYB10C:112-222aa), and $A c B H L H 42$. The amplified fragments were ligated to the $\mathrm{PGBKT7}$ vector. $\mathrm{AD}$ and $\mathrm{BD}$ constructs were co-transformed into yeast strain AH109 according to the manufacturer's instructions (SK2400-200T, Coolaber, Beijing, China). The co-transformants were initially selected on synthetic dropout medium lacking Leu and $\operatorname{Trp}(\mathrm{SD} /-\mathrm{Leu} /-\operatorname{Trp})$ and were replicated on quadruple-dropout medium deficient in Ade, His, Leu, and Trp (SD/-Ade/-His/-Leu/-Trp). They were then supplemented with $15 \mathrm{mM}$ 3-amino-1,2,4-triazole (3AT) CA1311 (Coolaber, Beijing, China), which is a competitive inhibitor of the HIS3 gene product. $X$ - $\alpha$-gal was also used to assess $\beta$-galactosidase activity and confirm positive interactions.

\section{Conclusions}

This study comprehensively analyzed R2R3-MYB TFs in the kiwifruit genome, demonstrating the synergistic effect of light and temperature on anthocyanin accumulation in kiwifruit. This study also showed that AcMYB10 and AcbHLH42 are interacting partners that contribute to anthocyanin biosynthesis by activating the transcription of AcLDOX and AcF3GT. Our study provides a basis for the functional study of R2R3-MYBs in kiwifruit, presenting new insights on the physiological mechanism and regulatory function of $A c M Y B 10$, as well as valuable knowledge that could be used to enhance cultivation practices and improve the quality of kiwifruit.

Supplementary Materials: Supplementary materials can be found at http://www.mdpi.com/1422-0067/20/20/ 5228/s1.

Author Contributions: Y.W. proposed the research topic and revised the article; M.Y. designed the study, analyzed and interpreted the data, and drafted the article. Y.M. revised the article. All authors read and approved the manuscript for publication.

Funding: This work was supported by the Natural Science Foundation of China (NSFC), project numbers 31501745, 30671433, and 31171945.

Conflicts of Interest: The authors declare no conflict of interest. 


\section{Abbreviations}

$\begin{array}{ll}\text { HMM } & \text { Hidden Markov Model } \\ \text { CDS } & \text { coding sequences } \\ \text { FPKM } & \text { Fragments per kilobase of transcript per million mapped reads } \\ \text { VIGS } & \text { Virus-induced gene silencing } \\ \text { qRT-PCR } & \text { Quantitative real-time PCR } \\ \text { DAP } & \text { days after pollination } \\ \text { YFP } & \text { yellow fluorescent protein } \\ \text { RFP } & \text { red fluorescent protein } \\ \text { 3AT } & \text { 3-amino-1,2,4-triazole } \\ \text { CRE } & \text { Cis-acting regulatory element } \\ \text { LTR } & \text { Low-temperature stress } \\ \text { MRE } & \text { MYB-recognizing element }\end{array}$

\section{References}

1. Feller, A.; Machemer, K.; Braun, E.L.; Grotewold, E. Evolutionary and comparative analysis of MYB and bHLH plant transcription factors. Plant J. 2011, 66, 94-116. [CrossRef] [PubMed]

2. Jin, H.L.; Martin, C. Multifunctionality and diversity within the plant MYB-gene family. Plant Mol. Biol. 1999, 41, 577-585. [CrossRef] [PubMed]

3. Stracke, R.; Werber, M.; Weisshaar, B. The R2R3-MYB gene family in Arabidopsis thaliana. Curr. Opin. Plant Biol. 2001, 4, 447-456. [CrossRef]

4. Du, H.; Yang, S.-S.; Liang, Z.; Feng, B.-R.; Liu, L.; Huang, Y.-B.; Tang, Y.-X. Genome-wide analysis of the MYB transcription factor superfamily in soybean. BMC Plant Biol. 2012, 12, 106. [CrossRef]

5. Katiyar, A.; Smita, S.; Lenka, S.K.; Rajwanshi, R.; Chinnusamy, V.; Bansal, K.C. Genome-wide classification and expression analysis of MYB transcription factor families in rice and Arabidopsis. BMC Genom. 2012, 13, 544. [CrossRef]

6. Pazares, J.; Ghosal, D.; Wienand, U.; Peterson, P.A.; Saedler, H. The regulatory c1 locus of Zea-mays encodes a protein with homology to myb protooncogene products and with structural similarities to transcriptional activators. Embo J. 1987, 6, 3553-3558. [CrossRef]

7. Gonzalez, A.; Zhao, M.; Leavitt, J.M.; Lloyd, A.M. Regulation of the anthocyanin biosynthetic pathway by the TTG1/bHLH/Myb transcriptional complex in Arabidopsis seedlings. Plant J. 2008, 53, 814-827. [CrossRef]

8. Deluc, L.; Bogs, J.; Walker, A.R.; Ferrier, T.; Decendit, A.; Merillon, J.-M.; Robinson, S.P.; Barrieu, F. The transcription factor VvMYB5b contributes to the regulation of anthocyanin and proanthocyanidin biosynthesis in developing grape berries. Plant Physiol. 2008, 147, 2041-2053. [CrossRef]

9. Feng, S.; Wang, Y.; Yang, S.; Xu, Y.; Chen, X. Anthocyanin biosynthesis in pears is regulated by a R2R3-MYB transcription factor PyMYB10. Planta 2010, 232, 245-255. [CrossRef]

10. Espley, R.V.; Hellens, R.P.; Putterill, J.; Stevenson, D.E.; Kutty-Amma, S.; Allan, A.C. Red colouration in apple fruit is due to the activity of the MYB transcription factor, MdMYB10. Plant J. 2007, 49, 414-427. [CrossRef]

11. Li, J.; Li, X.; Soejarto, D. Actinidiaceae. Flora China 2007, 12, 334-360.

12. Montefiori, M.; Comeskey, D.J.; Wohlers, M.; McGhie, T.K. Characterization and Quantification of Anthocyanins in Red Kiwifruit (Actinidia spp.). J. Agric. Food Chem. 2009, 57, 6856-6861. [CrossRef] [PubMed]

13. Jaeger, S.R.; Harker, F.R. Consumer evaluation of novel kiwifruit: Willingness-to-pay. J. Sci. Food Agric. 2005, 85, 2519-2526. [CrossRef]

14. Yousuf, B.; Gul, K.; Wani, A.A.; Singh, P. Health Benefits of Anthocyanins and Their Encapsulation for Potential Use in Food Systems: A Review. Crit. Rev. Food Sci. Nutr. 2016, 56, 2223-2230. [CrossRef]

15. Montefiori, M.; Espley, R.V.; Stevenson, D.; Cooney, J.; Datson, P.M.; Saiz, A.; Atkinson, R.G.; Hellens, R.P.; Allan, A.C. Identification and characterisation of F3GT1 and F3GGT1, two glycosyltransferases responsible for anthocyanin biosynthesis in red-fleshed kiwifruit (Actinidia chinensis). Plant J. 2011, 65, 106-118. [CrossRef] 
16. Fraser, L.G.; Seal, A.G.; Montefiori, M.; McGhie, T.K.; Tsang, G.K.; Datson, P.M.; Hilario, E.; Marsh, H.E.; Dunn, J.K.; Hellens, R.P.; et al. An R2R3 MYB transcription factor determines red petal colour in an Actinidia (kiwifruit) hybrid population. BMC Genom. 2013, 14, 28. [CrossRef]

17. Li, W.; Ding, Z.; Ruan, M.; Yu, X.; Peng, M.; Liu, Y. Kiwifruit R2R3-MYB transcription factors and contribution of the novel AcMYB75 to red kiwifruit anthocyanin biosynthesis. Sci. Rep. 2017, 7, 16861. [CrossRef]

18. Liu, Y.; Zhou, B.; Qi, Y.; Chen, X.; Liu, C.; Liu, Z.; Ren, X. Expression Differences of Pigment Structural Genes and Transcription Factors Explain Flesh Coloration in Three Contrasting Kiwifruit Cultivars. Front. Plant Sci. 2017, 8, 1507. [CrossRef]

19. Wang, L.; Tang, W.; Hu, Y.; Zhang, Y.; Sun, J.; Guo, X.; Lu, H.; Yang, Y.; Fang, C.; Niu, X.; et al. A MYB/bHLH complex regulates tissue-specific anthocyanin biosynthesis in the inner pericarp of red-centered kiwifruit Actinidia chinensis cv. Hongyang. Plant J. Cell Mol. Biol. 2019, 99, 359-378. [CrossRef]

20. Jaakola, L. New insights into the regulation of anthocyanin biosynthesis in fruits. Trends Plant Sci. 2013, 18, 477-483. [CrossRef]

21. Cominelli, E.; Gusmaroli, G.; Allegra, D.; Galbiati, M.; Wade, H.K.; Jenkins, G.I.; Tonelli, C. Expression analysis of anthocyanin regulatory genes in response to different light qualities in Arabidopsis thaliana. J. Plant Physiol. 2008, 165, 886-894. [CrossRef] [PubMed]

22. Bai, S.; Sun, Y.; Qian, M.; Yang, F.; Ni, J.; Tao, R.; Li, L.; Shu, Q.; Zhang, D.; Teng, Y. Transcriptome analysis of bagging-treated red Chinese sand pear peels reveals light-responsive pathway functions in anthocyanin accumulation. Sci. Rep. 2017, 7, 63. [CrossRef] [PubMed]

23. Jiang, M.; Ren, L.; Lian, H.; Liu, Y.; Chen, H. Novel insight into the mechanism underlying light-controlled anthocyanin accumulation in eggplant (Solanum melongena L.). Plant Sci. 2016, 249, 46-58. [CrossRef] [PubMed]

24. Lai, B.; Li, X.-J.; Hu, B.; Qin, Y.-H.; Huang, X.-M.; Wang, H.-C.; Hu, G.-B. LcMYB1 Is a Key Determinant of Differential Anthocyanin Accumulation among Genotypes, Tissues, Developmental Phases and ABA and Light Stimuli in Litchi chinensis. PloS ONE 2014, 9, e86293. [CrossRef]

25. Niu, S.-S.; Xu, C.-J.; Zhang, W.-S.; Zhang, B.; Li, X.; Lin-Wang, K.; Ferguson, I.B.; Allan, A.C.; Chen, K.-S. Coordinated regulation of anthocyanin biosynthesis in Chinese bayberry (Myrica rubra) fruit by a R2R3 MYB transcription factor. Planta 2010, 231, 887-899. [CrossRef]

26. Takos, A.M.; Jaffe, F.W.; Jacob, S.R.; Bogs, J.; Robinson, S.P.; Walker, A.R. Light-induced expression of a MYB gene regulates anthocyanin biosynthesis in red apples. Plant Physiol. 2006, 142, 1216-1232. [CrossRef]

27. Azuma, A.; Yakushiji, H.; Koshita, Y.; Kobayashi, S. Flavonoid biosynthesis-related genes in grape skin are differentially regulated by temperature and light conditions. Planta 2012, 236, 1067-1080. [CrossRef]

28. Lin-Wang, K.; Micheletti, D.; Palmer, J.; Volz, R.; Lozano, L.; Espley, R.; Hellens, R.P.; Chagne, D.; Rowan, D.D.; Troggio, M.; et al. High temperature reduces apple fruit colour via modulation of the anthocyanin regulatory complex. Plant Cell Environ. 2011, 34, 1176-1190. [CrossRef]

29. Wang, N.; Zhang, Z.; Jiang, S.; Xu, H.; Wang, Y.; Feng, S.; Chen, X. Synergistic effects of light and temperature on anthocyanin biosynthesis in callus cultures of red-fleshed apple (Malus sieversii $\mathrm{f}$. niedzwetzkyana). Plant Cell Tissue Organ. Cult. 2016, 127, 217-227. [CrossRef]

30. Li, B.; Xia, Y.; Wang, Y.; Qin, G.; Tian, S. Characterization of Genes Encoding Key Enzymes Involved in Anthocyanin Metabolism of Kiwifruit during Storage Period. Front. Plant Sci. 2017, 8, 341. [CrossRef]

31. Man, Y.-P.; Wang, Y.-C.; Li, Z.-Z.; Jiang, Z.-W.; Yang, H.-L.; Gong, J.-J.; He, S.-S.; Wu, S.-Q.; Yang, Z.-Q.; Zheng, J.; et al. High-temperature inhibition of biosynthesis and transportation of anthocyanins results in the poor red coloration in red-fleshed Actinidia chinensis. Physiol. Plant 2015, 153, 565-583. [CrossRef] [PubMed]

32. Dubos, C.; Stracke, R.; Grotewold, E.; Weisshaar, B.; Martin, C.; Lepiniec, L. MYB transcription factors in Arabidopsis. Trends Plant Sci. 2010, 15, 573-581. [CrossRef] [PubMed]

33. Yamane, T.; Jeong, S.T.; Goto-Yamamoto, N.; Koshita, Y.; Kobayashi, S. Effects of temperature on anthocyanin biosynthesis in grape berry skins. Am. J. Enol. Vitic. 2006, 57, 54-59.

34. Mohanty, S.; Grimm, B.; Tripathy, B.C. Light and dark modulation of chlorophyll biosynthetic genes in response to temperature. Planta 2006, 224, 692-699. [CrossRef] [PubMed] 
35. Cox, K.A.; McGhie, T.K.; White, A.; Woolf, A.B. Skin colour and pigment changes during ripening of 'Haas' avocado fruit. Postharvest Biol. Technol. 2004, 31, 287-294. [CrossRef]

36. Lee, J.; He, K.; Stolc, V.; Lee, H.; Figueroa, P.; Gao, Y.; Tongprasit, W.; Zhao, H.; Lee, I.; Deng, X. Analysis of transcription factor HY5 genomic binding sites revealed its hierarchical role in light regulation of development. Plant Cell 2007, 19, 731-749. [CrossRef]

37. Zimmermann, I.M.; Heim, M.A.; Weisshaar, B.; Uhrig, J.F. Comprehensive identification of Arabidopsis thaliana MYB transcription factors interacting with R/B-like BHLH proteins. Plant J. 2004, 40, 22-34. [CrossRef]

38. Li, J.G.; Yang, X.Y.; Wang, Y.; Li, X.J.; Gao, Z.F.; Pei, M.; Chen, Z.L.; Qu, L.J.; Gu, H.Y. Two groups of MYB transcription factors share a motif which enhances trans-activation activity. Biochem. Biophys. Res. Commun. 2006, 341, 1155-1163. [CrossRef]

39. Chen, W.; Gong, L.; Guo, Z.; Wang, W.; Zhang, H.; Liu, X.; Yu, S.; Xiong, L.; Luo, J. A novel integrated method for large-scale detection, identification, and quantification of widely targeted metabolites: Application in the study of rice metabolomics. Mol. Plant 2013, 6, 1769-1780. [CrossRef]

40. Sainz, M.B.; Grotewold, E.; Chandler, V.L. Evidence for direct activation of an anthocyanin promoter by the maize C1 protein and comparison of DNA binding by related Myb domain proteins. Plant Cell 1997, 9, 611-625. [CrossRef]

41. Zhu, Z.; Wang, H.; Wang, Y.; Guan, S.; Wang, F.; Tang, J.; Zhang, R.; Xie, L.; Lu, Y. Characterization of the cis elements in the proximal promoter regions of the anthocyanin pathway genes reveals a common regulatory logic that governs pathway regulation. J. Exp. Bot. 2015, 66, 3775-3789. [CrossRef] [PubMed]

42. Yuan, Y. Research Progress of Kiwifruit in Tissue Culture. In Proceedings of the 2011 International Conference on Future Computer Science and Application (FCSA 2011), Hongkong, China, 18-19 June 2011; pp. 17-20.

43. Akbas, F.; Isikalan, C.; Namli, S. Callus Induction and Plant Regeneration from Different Explants of Actinidia deliciosa. Appl. Biochem. Biotechnol. 2009, 158, 470-475. [CrossRef] [PubMed]

44. Pilkington, S.M.; Crowhurst, R.; Hilario, E.; Nardozza, S.; Fraser, L.; Peng, Y.; Gunaseelan, K.; Simpson, R.; Tahir, J.; Deroles, S.C.; et al. A manually annotated Actinidia chinensis var. chinensis (kiwifruit) genome highlights the challenges associated with draft genomes and gene prediction in plants. BMC Genom. 2018, 19, 257. [CrossRef] [PubMed]

45. Kumar, S.; Stecher, G.; Tamura, K. MEGA7: Molecular Evolutionary Genetics Analysis Version 7.0 for Bigger Datasets. Mol. Biol. Evol. 2016, 33, 1870-1874. [CrossRef] [PubMed]

46. Petriccione, M.; Mastrobuoni, F.; Zampella, L.; Scortichini, M. Reference gene selection for normalization of RT-qPCR gene expression data from Actinidia deliciosa leaves infected with Pseudomonas syringae pv. actinidiae. Sci. Rep. 2015, 5, 16961. [CrossRef]

47. Shin, J.; Park, E.; Choi, G. PIF3 regulates anthocyanin biosynthesis in an HY5-dependent manner with both factors directly binding anthocyanin biosynthetic gene promoters in Arabidopsis. Plant J. 2007, 49, 981-994. [CrossRef]

48. Lim, S.H.; Kim, D.H.; Kim, J.K.; Lee, J.Y.; Ha, S.H. A Radish Basic Helix-Loop-Helix Transcription Factor, RsTT8 Acts a Positive Regulator for Anthocyanin Biosynthesis. Front. Plant Sci. 2017, 8, 1917. [CrossRef]

49. Hiscox, J.D.; Israelstam, G.F. Method for the extraction of chlorophyll from leaf tissue without maceration. Can. J. Bot. 1979, 57, 1332-1334. [CrossRef]

50. Wang, A.; Li, R.; Ren, L.; Gao, X.; Zhang, Y.; Ma, Z.; Ma, D.; Luo, Y. A comparative metabolomics study of flavonoids in sweet potato with different flesh colors (Ipomoea batatas (L.) Lam). Food Chem. 2018, 260, 124-134. [CrossRef]

51. Hellens, R.P.; Allan, A.C.; Friel, E.N.; Bolitho, K.; Grafton, K.; Templeton, M.D.; Karunairetnam, S.; Gleave, A.P.; Laing, W.A. Transient expression vectors for functional genomics, quantification of promoter activity and RNA silencing in plants. Plant Methods 2005, 1, 13. [CrossRef]

52. Palapol, Y.; Ketsa, S.; Kui, L.-W.; Ferguson, I.B.; Allan, A.C. A MYB transcription factor regulates anthocyanin biosynthesis in mangosteen (Garcinia mangostana L.) fruit during ripening. Planta 2009, 229, 1323-1334. [CrossRef] [PubMed]

53. Liu, Y.; Zhou, B.; Qi, Y.; Liu, C.; Liu, Z.; Ren, X. Biochemical and functional characterization of AcUFGT3a, a galactosyltransferase involved in anthocyanin biosynthesis in the red-fleshed kiwifruit (Actinidia chinensis). Physiol. Plant 2018, 162, 409-426. [CrossRef] [PubMed] 
54. Wang, T.; Atkinson, R.; Janssen, B. The choice of Agrobacterium strain for transformation of kiwifruit. Acta Hortic 2007, 753, 227-232. [CrossRef]

55. Kumar, K.R.R.; Kirti, P.B. A mitogen-activated protein kinase, AhMPK6 from peanut localizes to the nucleus and also induces defense responses upon transient expression in tobacco. Plant Physiol. Biochem. 2010, 48, 481-486. [CrossRef] article distributed under the terms and conditions of the Creative Commons Attribution (CC BY) license (http://creativecommons.org/licenses/by/4.0/). 